\title{
Nonlinear Dynamics of a Power-Split Transmission Unit with a Planetary Gear Train and Selectable One-Way Clutches
}

\author{
Zhizhou Jia (iD) and Pingkang Li \\ School of Mechanical, Electronic and Control Engineering, Beijing Jiaotong University, No. 3 Shangyuancun, Haidian District, \\ Beijing 100044, China
}

Correspondence should be addressed to Zhizhou Jia; zz_jia@bjtu.edu.cn

Received 25 September 2019; Revised 31 January 2020; Accepted 20 February 2020; Published 30 March 2020

Academic Editor: Guang Li

Copyright $(92020$ Zhizhou Jia and Pingkang Li. This is an open access article distributed under the Creative Commons Attribution License, which permits unrestricted use, distribution, and reproduction in any medium, provided the original work is properly cited.

\begin{abstract}
A Planetary Gear Train (PGT) can be used in series-parallel transmission to redistribute powers. Selectable one-way clutches (SOWCs), compared with traditional friction clutches, can simplify controls and diversify patterns especially for hybrid transmissions. In this paper, a nonlinear torsional model of a power-split PGT coupled with three SOWCs is proposed. Piecewise nonlinearities of SOWCs as well as clearance, time-varying mesh stiffness, and synthetic transmission error of the PGT are considered. With a specified group of comprehensive evaluation indices, influences of piecewise nonlinearities of SOWCs are explored. Simulation results show that the piecewise nonlinearities of SOWCs can diminish collision range and reduce resonances of the PGT. Further research studies on parameter configurations of each SOWC reveal that of the three SOWCs, stiffness and radius of the SOWC connected to the sun gear of the PGT are dominant factors. Large stiffness and effective radius of the SOWC render the PGT fall into chaos on lower meshing frequencies; however, enormous impact vibrations occur to the SOWC if it gets too soft. Additionally, the increase of the damping ratio of the SOWC connected to sun gear can distinctly reduce the vibration and maximum dynamic load of the system on the entire working range.
\end{abstract}

\section{Introduction}

Friction clutches, as common executive mechanisms, are widely used in vehicle transmissions. Features of the friction engagement governed by diaphragm springs in general, and problems such as friction, wear, temperature, sliding speed, and contact pressure [1-3] in particular, have rendered high energy consumption, control complexity, and slow response speed that prevent the existing vehicle transmissions from upgrading.

One-way clutches (OWCs), in contrast, have the superiorities of simpler structure, faster response speed, and lower energy consumption. To resolve the defect of single direction transmission of OWCs, controllable transformations, which add assembly elements to manage the trigger of engagement, reinforce SOWCs by providing options of transferring torque in neither direction, either direction, or both directions. Manufacturers such as
General Motors [4], Ford [5], Toyota [6], Magna [7], Allison Transmission [8], and Means Industries [9] owned large amount of recent granted patents related to SOWCs. Samie et al. [10] applied a SOWC in a 6-speed automatic transmission and demonstrated its potential capabilities to save cost, cut weight, and improve fuel economy. Bird et al. [11] proposed an electrical SOWC in a hybrid transmission and introduced challenges and benefits associated with SOWC. Zhao et al. [12] designed an uninterrupted gearshift transmission with SOWCs to alleviated shocks or jerks in shift process. It is reasonable to infer that SOWCs will play important roles in future vehicle transmissions.

Associated with the trend of applications, piecewise nonlinearities of SOWCs and their influences on dynamics call for more attention. Research studies on belt-pulley systems [13-15] have demonstrated OWCs' potential capabilities of reducing noises and vibrations. As key 
ingredients of a transmission, gears also suffer from multiple nonlinearities. Though plenty of research studies have been published on nonlinear dynamics of PGT [16-20], most of them have one element of the PGT fixed to construct a single-input single-output transmission, and OWCs are not commonly considered because of their application limitations in the past days. Gill-Jeong [21] validated OWCs' function of reducing vibration and pointed out that stiffness of the OWC had great effects on the behavior of a gear pair. Liu et al. [22] concluded that the system was prone to involve chaotic motions with higher excitation frequencies and larger torsional stiffness of OWCs. However, whether these results are consecutive with PGT is not clear. Nonlinear dynamics of PGT with piecewise nonlinearities of SOWCs under the influences of floating sun gear, floating planet carrier, and floating ring gear of the PGT are unknown. Additionally, attenuation of torsional oscillations is important in automotive powertrains [23-26]. Optimal design of the stiffness and damping of a clutch is one method to reduce torsional oscillations [27-29]. Nevertheless, those research studies focus mainly on friction clutches. Parameter configurations of SOWCs and their influences on torsional oscillations need further research.

In the series-parallel transsion, PGT is the key component to redistribute powers. Combination of the power-split PGT with SOWCs empowers the transmission system with more flexibilities. However, multiple nonlinearities of PGT and SOWCs render complex dynamic behaviors that greatly affect the transmission quality and reliability. Although several research studies have been proposed with gears and OWCs, the dynamic behaviors of PGT with three SWOCs under the influences of the floating ring gear, sun gear, and carrier of the PGT are not clear. Parameter influences of each SOWC are unknown. This paper establishes a nonlinear torsional model of a two DOF singlestage PGT coupled with three SOWCs. Gear clearance, time-varying mesh stiffness, synthetic transmission error, and piecewise characteristic of SOWCs are considered. To better analyze the system, comprehensive evaluation indices from bifurcation, vibration, load, and collision aspects are drawn. Nonlinear characteristics of the system and parameter configuration impacts of each SOWC are analyzed. Complex dynamic behaviors are revealed. The research results can be used to guide the parameter configurations of SOWCs.

\section{Modeling of the Transmission Unit}

When a single-stage PGT with a sun gear, a ring gear, and a carrier is to be connected into a transmission, after the input and the output are chosen, the remaining element can either be fixed, which forms a fixed ratio transmission, or connected to a controlled object, which forms a power-split transmission. In the classic power-split structure of a PGT, the carrier is connected to the main input; the ring gear is connected to the main output; and the sun gear serves as either input terminal or output terminal to balance the lever inside the PGT and the power demand of the system.
Meanwhile, another power source should be connected to the ring gear in series to form a series-parallel structure. This configuration decouples the movement of the main input from the main output and is flexible with dynamic power allocation. The transmission unit proposed in this paper introduces three SOWCs in a power-split transmission. The three SOWCs are connected to the sun gear of the PGT (SOWC-S), the carrier of the PGT (SOWC-C), and the ring gear of the PGT (SOWC-R) respectively, as shown in Figure 1.

SOWC can insulate the transmission from unwelcomed rotation direction and switch between models of fixed ratio transmission and power-split transmission. In the powersplit series-parallel structure, SOWC-R can separate the system into two subsystems by fixing the ring gear and setting free the other terminal. A graded recovery of energy can also be achieved by fixing the carrier and choosing to actuate one or more generators. This paper focuses on the power-split model and the following assumptions are specified:

(1) Each SOWC and all components of the PGT, including a ring gear, a sun gear, a carrier and $i$ planet gears, are assumed to rotate only in $\theta$ direction (positive clockwise). Hence, the dynamic model has $6+i$ degrees of freedom.

(2) Bodies of the gears and the connections between gear and SOWC are considered rigid.

(3) The carrier is defined to rotate only in clockwise direction.

Symbols are introduced as follows. $S, R$, and $C$ represent the sun gear, the ring gear, and the carrier, respectively. $P i$ stands for the $i$ th planet gear, where $i=1,2,3$. $O C-p$ stands for the SOWC connected with $p$, where $p=S$, $R$, and C. $P C$ stands for the combination of the carrier and 3 planet gears. $Z$ is the number of teeth. $S P i$ and $R P i$ represent corresponding physical quantity between $S$ and $P i$ and $R$ and $P i$, respectively. For physical quantities, $I$ is rotational inertia. $m$ is equivalent mass. $\theta$ is angular displacement. $u$ stands for the absolute displacement, where $u_{j}=\theta_{j} \cdot r_{b j}$, and $r_{b}$ is the effective radius, $j=S, P i, R, C, O C-p$. $F$ is the effective force related to torque $T$ and effective radius. $\omega$ is rotational speed. $\omega_{m}$ is the rotational speed of mesh that correlates to the mesh frequency $f_{m} . k$ is stiffness. $c$ is damping. $t$ is time. Schematic representation of the power-split PGT is shown in Figure 2, where gear clearance, time-varying mesh stiffness, and synthetic transmission error are considered.

Relative displacements of PGT, which are also the dynamic transmission errors (DTEs), are defined as follows:

$$
\left\{\begin{array}{l}
u_{S P i}=\left(\theta_{C}-\theta_{S}\right) \cdot r_{S}-u_{P i}-e_{S P i}, \\
u_{R P i}=u_{P i}-\left(\theta_{R}-\theta_{C}\right) \cdot r_{R}-e_{R P i},
\end{array}\right.
$$

where $e_{q}(t)$ is synthetic transmission error and $q=S P i, R P i$. The machining error and assembly error cause a contour deviation from the theoretical position; hence, a displacement excitation is generated. Assuming this excitation is 


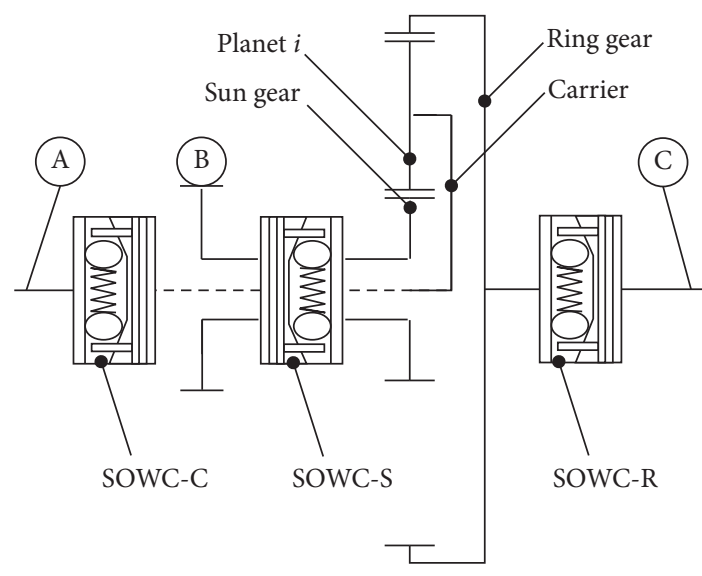

Figure 1: Configurations of the transmission unit.

related with $\omega_{m}$ and has a phase of $\varphi_{e q}$, the synthetic transmission error is

$$
e_{q}(t)=e_{q} \cos \left(\omega_{m} t+\varphi_{e q}\right) .
$$

As shown in Figure 2, a gear clearance of $2 b$ is considered (assuming that $b_{S P i}=b_{R P i}=b$ ). Therefore, a nonlinear function of $g(\cdot)$ is used to describe the relationship of gear mesh displacement with relative displacement $u$ and backlash $2 b$ :

$$
g(u)= \begin{cases}u-b, & u>b, \\ 0, & |u| \leq b \\ u+b, & u<-b .\end{cases}
$$

Since the number of teeth that involves in the transmission varies with time, a time-varying mesh stiffness must be considered. Based on the research studies of [30, 31], in this paper, the time-varying mesh stiffness is defined as

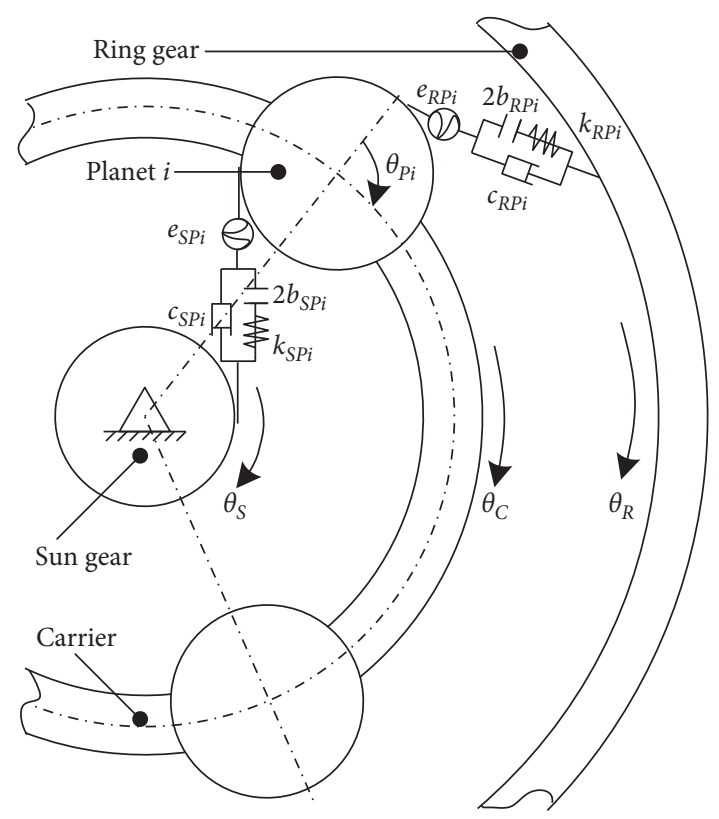

FIGURE 2: Schematic representation of the power-split PGT.

$$
\begin{aligned}
k_{q}(t)= & k_{a q}\left(1+\sum_{s=1}^{N}\left(A_{s} \cos \left(s \omega_{m} t-\varphi_{k q}\right)\right.\right. \\
& \left.\left.+B_{s} \sin \left(s \omega_{m} t-\varphi_{k q}\right)\right)\right),
\end{aligned}
$$

where

$A_{s}=\left(1 / s \pi \varepsilon_{q}\right) \sin \left(2 \pi s \varepsilon_{q}\right) ; B_{s}=\left(1 / s \pi \varepsilon_{q}\right)\left(1-\cos \left(2 \pi s \varepsilon_{q}\right)\right) ; k_{a q}$ is the average mesh stiffness; $\varphi_{k q}$ is mesh phase; $\varepsilon_{q}$ is contact ratio; and $q=S P i, R P i$.

The equations of motion for the coupled system can be governed by

$$
\left\{\begin{array}{l}
I_{S} \ddot{\theta}_{S}=\sum_{i=1}^{3} k_{S P i}(t) g\left(u_{S P i}\right) r_{b S}+\sum_{i=1}^{3} c_{S P i} \dot{u}_{S P i} r_{b S}-T_{O C-S}, \\
I_{P i} \ddot{\theta}_{P i}=k_{S P i}(t) g\left(u_{S P i}\right) r_{b P i}+c_{S P i} \dot{u}_{S P i} r_{b P i}-k_{R P i}(t) g\left(u_{R P i}\right) r_{b P i}-c_{R P i} \dot{u}_{R P i} r_{b P i}, \\
I_{P C} \ddot{\theta}_{C}=T_{O C-C}-\sum_{i=1}^{3} k_{S P i}(t) g\left(u_{S P i}\right) r_{b C}-\sum_{i=1}^{3} c_{S P i} \dot{u}_{S P i} r_{b C}-\sum_{i=1}^{3} k_{R P i}(t) g\left(u_{R P i}\right) r_{b C}-\sum_{i=1}^{3} c_{R P i} \dot{u}_{R P i} r_{b C}, \\
\ddot{I}_{R} \ddot{\theta}_{R}=\sum_{i=1}^{3} k_{R P i}(t) g\left(u_{R P i}\right) r_{b R}+\sum_{i=1}^{3} c_{R P i} \dot{u}_{R P i} r_{b R}-T_{O C-R}, \\
I_{O C-C} \ddot{\theta}_{O C-C}=T_{1}-T_{O C-C}, \\
I_{O C-S} \ddot{\theta}_{O C-S}=T_{O C-S}-T_{2}, \\
I_{O C-R} \ddot{\theta}_{O C-R}=T_{O C-R}-T_{3},
\end{array}\right.
$$

where $\quad c_{S P i}=2 \zeta_{S P i} \sqrt{m_{S} m_{P}} \quad k_{a S P i} /\left(m_{S}+m_{P}\right) ; c_{R P i}=2 \zeta_{R P i}$ $\sqrt{m_{R} m_{P} k_{a R P i} /\left(m_{R}+m_{P}\right)} ; \zeta_{S P i}$ and $\zeta_{R P i}$ are damping ratios; $I_{P C}=I_{C}+\sum_{i=0}^{3} m_{P i} r_{b C}^{2} ; T_{O C-C}, T_{O C-S}$, and $T_{O C-R}$ stand for the math model for each SOWC; and $T_{1}$ is the input, $T_{2}$ is the output, and $T_{3}$ can either input power or output power depending on the sun gear's rotation direction. 


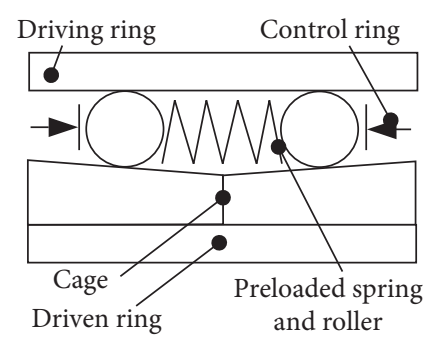

(a)

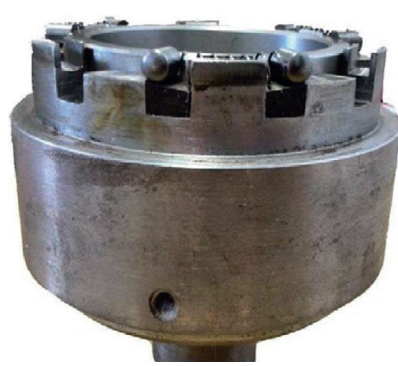

(b)

Figure 3: The roller type SOWC. (a) Schematic diagram. (b) Actual structure.

Schematic diagram of the proposed SOWC is illustrated in Figure 3(a). It uses self-locking principle of friction to achieve torque transmissions. The preloaded spring is used to diminish idle angle during model switch. Different from traditional roller type OWC, it transforms radial arrangement into axial arrangement and adds control ring to select transmission directions. The actual structure is shown in Figure 3(b).

When working under a stable environment of a specific one-way model, a SOWC is a OWC. It can be governed using a nonlinear spring-damper model:

$$
T_{\mathrm{OC}}=k_{\mathrm{OC}} f_{c}(\Delta \theta)+c_{\mathrm{OC}} \dot{f}_{c}(\Delta \theta) \text {, }
$$

where $c_{O C}=2 \zeta_{O C} \sqrt{k_{O C} I_{O C}} ; \zeta_{O C}$ stands for the damping ratio of SOWC; and $f_{c}(\cdot)$ is used to describe the piecewise nonlinearity of SOWC.

$$
f_{c}(\Delta \theta)= \begin{cases}\Delta \theta, & \Delta \theta>0, \\ 0, & \Delta \theta \leq 0,\end{cases}
$$

and for each SOWC,

$$
\left\{\begin{array}{l}
\Delta \theta_{C O C}=\theta_{O C-C}-\theta_{C}, \\
\Delta \theta_{S O C}=\theta_{S}-\theta_{O C-S}, \\
\Delta \theta_{R O C}=\theta_{R}-\theta_{O C-R} .
\end{array}\right.
$$

To deal with the enormous differences in magnitude of physical quantities, define characteristic length $b_{a}$ and characteristic frequency $\omega_{a}$. Setting $b_{a}=1 e-6 \mathrm{~m}$ and $\omega_{a}=\sqrt{k_{a S P i}\left(m_{S}+m_{P}\right) / m_{S} m_{P}}$, the relationships can be described as

$$
\left\{\begin{array}{l}
u_{j}=\delta_{j} b_{a}, \\
\tau=t \omega_{a} .
\end{array}\right.
$$

The dimensionless model is

$$
\left\{\begin{array}{l}
\ddot{\delta}_{S}=\sum_{i=1}^{3}\left(\kappa_{S P i}(\tau) G\left(\delta_{S P i}\right)+\xi_{S P i} \dot{\delta}_{S P i}\right)-\frac{m_{O C-S}}{m_{S}}\left(\kappa_{O C-S} f_{c}\left(\Delta \delta_{S O C}\right)+\xi_{O C-S} \dot{f}_{c}\left(\Delta \delta_{S O C}\right)\right), \\
\ddot{\delta}_{P i}=\frac{m_{S}}{m_{P i}}\left(\kappa_{S P i}(\tau) G\left(\delta_{S P i}\right)+\xi_{S P i} \dot{\delta}_{S P i}\right)-\frac{m_{R}}{m_{P i}}\left(\kappa_{R P i}(\tau) G\left(\delta_{R P i}\right)+\xi_{R P i} \dot{\delta}_{R P i}\right), \\
\ddot{\delta}_{C}=-\frac{m_{S}}{m_{P C}} \sum_{i=1}^{3}\left(\kappa_{S P i}(\tau) G\left(\delta_{S P i}\right)+\xi_{S P i} \dot{\delta}_{S P i}\right)-\frac{m_{R}}{m_{P C}} \sum_{i=1}^{3}\left(\kappa_{R P i}(\tau) G\left(\delta_{R P i}\right)+\xi_{R P i} \dot{\delta}_{R P i}\right)+\frac{m_{O C-C}}{m_{P C}}\left(\kappa_{O C-C} f_{c}\left(\Delta \delta_{C O C}\right)+\xi_{O C-C} \dot{f}_{c}\left(\Delta \dot{\delta}_{C O C}\right)\right), \\
\ddot{\delta}_{R}=\sum_{i=1}^{3}\left(\kappa_{R P i}(\tau) G\left(\delta_{R P i}\right)+\xi_{R P i} \dot{\delta}_{R P i}\right)-\frac{m_{O C-R}}{m_{R}}\left(\kappa_{O C-R} f_{c}\left(\Delta \delta_{R O C}\right)+\xi_{O C-R} \dot{f}_{c}\left(\Delta \dot{\delta}_{R O C}\right)\right), \\
\ddot{\delta}_{O C-C}=-\kappa_{O C-C} f_{c}\left(\Delta \delta_{C O C}\right)-\xi_{O C-C} \dot{f}_{c}\left(\Delta \dot{\delta}_{C O C}\right)+P_{1}, \\
\ddot{\delta}_{O C-S}=\kappa_{O C-S} f_{c}\left(\Delta \delta_{S O C}\right)+\xi_{O C-S} \dot{f}_{c}\left(\Delta \dot{\delta}_{S O C}\right)-P_{2}, \\
\ddot{\delta}_{O C-R}=\kappa_{O C-R} f_{c}\left(\Delta \delta_{R O C}\right)+\xi_{O C-R} \dot{f}_{c}\left(\Delta \dot{\delta}_{R O C}\right)-P_{3},
\end{array}\right.
$$


TABle 1: Parameters of the system.

\begin{tabular}{lccc}
\hline Parameter & Value & Parameter & Value \\
\hline$Z_{S}$ & 15 & $\zeta_{S P i}=\zeta_{R P i}$ & 0.02 \\
$Z_{R}$ & 51 & $k_{a S P i}$ & $2.8783 e 8 \mathrm{~N} / \mathrm{m}$ \\
$Z_{P i}$ & 18 & $k_{a R P i}$ & $3.6050 e 8 \mathrm{~N} / \mathrm{m}$ \\
Pressure angle & $20 \mathrm{deg}$ & $b$ & $50 e-6 \mathrm{~m}$ \\
Module & $3 \mathrm{~mm}$ & $e$ & $20 e-6 \mathrm{~m}$ \\
$m_{S}$ & $0.10618 \mathrm{~kg}$ & $\zeta_{O C}$ & 0.01 \\
$m_{R}$ & $1.2274 \mathrm{~kg}$ & $k_{O C}$ & $1.50 \mathrm{e} 4 \mathrm{Nm} / \mathrm{rad}$ \\
$m_{P i}$ & $0.15289 \mathrm{~kg}$ & $m_{O C}$ & $0.10618 \mathrm{~kg}$ \\
\hline
\end{tabular}

where $\kappa_{S P i}(\tau)=k_{S P i}(t) / m_{S} \omega_{a}^{2}, \quad \kappa_{R P i}(\tau)=k_{R P i}(t) / m_{R} \omega_{a}^{2}$, $\xi_{S P i}=c_{S P i} / m_{S} \omega_{a}, \xi_{R P i}=c_{R P i} / m_{R} \omega_{a}, \kappa_{O C-C}=k_{O C-C} / m_{O C-C}$ $r_{b O C-C}^{2} \omega_{a}^{2}, \kappa_{O C-S}=k_{O C-S} / m_{O C-S} r_{b O C-S}^{2} \omega_{a}^{2}, \kappa_{O C-R}=k_{O C-R} /$ $m_{O C-R} r_{b O C-R}^{2} \omega_{a}^{2}, \quad \xi_{O C-C}=c_{O C-C} / m_{O C-C} r_{b O C-C}^{2} \omega_{a}, \quad \xi_{O C-S}=$ $c_{O C-S} / m_{O C-S} r_{b O C-S}^{2} \omega_{a}, \quad \xi_{O C-R}=c_{O C-R} / m_{O C-R} \quad r_{b O C-R}^{2} \omega_{a}$, $P_{1}=T_{1} / m_{O C-C} r_{b O C-C} b_{a} \omega_{a}^{2}, \quad P_{2}=T_{2} / m_{O C-S} r_{b O C-S} \quad b_{a} \omega_{a}^{2}$, $P_{3}=T_{3} / m_{O C-R} r_{b O C-R} b_{a} \omega_{a}^{2}, \Delta \delta_{C O C}=\delta_{O C-C}-\left(r_{b O C-C} / r_{b C}\right) \delta_{C}$, $\Delta \delta_{S O C}=\left(r_{b O C-S} / r_{b S}\right) \delta_{S}-\delta_{O C-S}, \Delta \delta_{R O C}=\left(r_{b O C-R} / r_{b R}\right) \delta_{R}-$ $\delta_{O C-R}$, and the nonlinear function

$$
G(\delta)= \begin{cases}\delta-\frac{b}{b_{a}}, & \delta>\frac{b}{b_{a}}, \\ 0, & |\delta| \leq \frac{b}{b_{a}}, \\ \delta+\frac{b}{b_{a}}, & \delta<-\frac{b}{b_{a}} .\end{cases}
$$

\section{Evaluation Indices and Nonlinear Dynamics}

Under the power-split model, speed control of the sun gear is essential to allocate power dynamically. Nevertheless, when the steady-state nonlinear dynamics are studied, meshing frequency defined by $f_{m}=Z_{P}\left|\omega_{P} / 2 \pi\right|$ is the key parameter to classify the unit. Considering that most power sources adopted in vehicle have output speeds lower than $8000 \mathrm{rpm}$, combined with the power-split working mode, the excitation frequencies are set below $1600 \mathrm{~Hz}$. Dynamic characteristics of the system are relatively simple on the range of $0-600 \mathrm{~Hz}$, and this paper focuses on a meshing frequency range of $600-1600 \mathrm{~Hz}$.

A specified group of comprehensive evaluation indices is firstly introduced in this section. Based on the indices, two cases are interweaved in the paper. To investigate the influences of SOWC on each position and avoid synchronies, three theoretically different SOWCs are used in Case A. They have same parameters except for their radius. Case B uses three identical SOWCs, which is more in accordance with the application, and their responses are used to determine parameters of SOWCs.

(i) Case A: $r_{b O C-S}=r_{b S}, r_{b O C-C}=r_{b C}, r_{b O C-R}=r_{b R}$.

(ii) Case B: $r_{b O C-S}=r_{b O C-C}=r_{b O C-R}=r_{b O C}$.

Parameters of the system are shown in Table 1. SOWCs are not adapted for heavy loads. For light vehicles, the maximum output torque for the engine is around $200 \mathrm{Nm}$. The input torque $T_{1}$ in the simulation is $60 \mathrm{Nm}$. This paper chooses a nominal stiffness of SOWC $15000 \mathrm{Nm} / \mathrm{rad}$. Nominal parameters of radius, stiffness, and damping of SOWCs may be proportionally changed in Section 4 to reveal parameters' effects of each SOWC.

3.1. Comprehensive Evaluation Indices. To better understand the system and evaluate dynamic performances, comprehensive evaluation indices from bifurcation, vibration, load, and collision aspects are specified.

3.1.1. Bifurcation Characteristics. Bifurcation theory is the mathematical study of changes in one parameter and the solutions of the differential equations. Bifurcation diagrams are applied to describe the structure and stability of the system as meshing frequency or radius of SOWCs changed.

3.1.2. Vibration Characteristics. Vibration characteristics of the system can be achieved by analyzing DTE. DTE of the PGT and SOWCs is given in equation (1) and (8), respectively. Root mean square of DTE (RMS-DTE),

$$
\text { RMS-DTE }=\sqrt{\frac{1}{N} \sum_{i=1}^{N}\left(\mathrm{DTE}_{i}\right)^{2}},
$$

and oscillating DTE (ODTE),

$$
\text { ODTE }=\sqrt{\frac{1}{N} \sum_{i=1}^{N}\left(\mathrm{DTE}_{i+1}-\mathrm{DTE}_{i}\right)^{2}},
$$

are used to describe the average transmission error and vibration situation, respectively.

3.1.3. Load Characteristics. Dynamic load coefficient is adopted to describe load characteristics, which is the ratio of maximum dynamic normal direction load $F_{n-d \max }$ and static normal direction load $F_{n-s}$.

$$
K_{v}=\frac{F_{n-d \max }}{F_{n-s}} .
$$


TABle 2: Discriminant of collisions.

\begin{tabular}{lccc}
\hline SOWC discriminant & SOWC state & PGT discriminant & PGT state \\
\hline$\Delta \theta_{\min } \geq 0$ & Noncollision & DTE $_{\min }>b$ & Noncollision \\
$\Delta \theta_{\min }<0$ & Collision & $\left|\mathrm{DTE}_{\min }\right| \leq b$ & Unilateral collision \\
- & - & DTE $_{\min }<-b$ & Bilateral collision \\
\hline
\end{tabular}

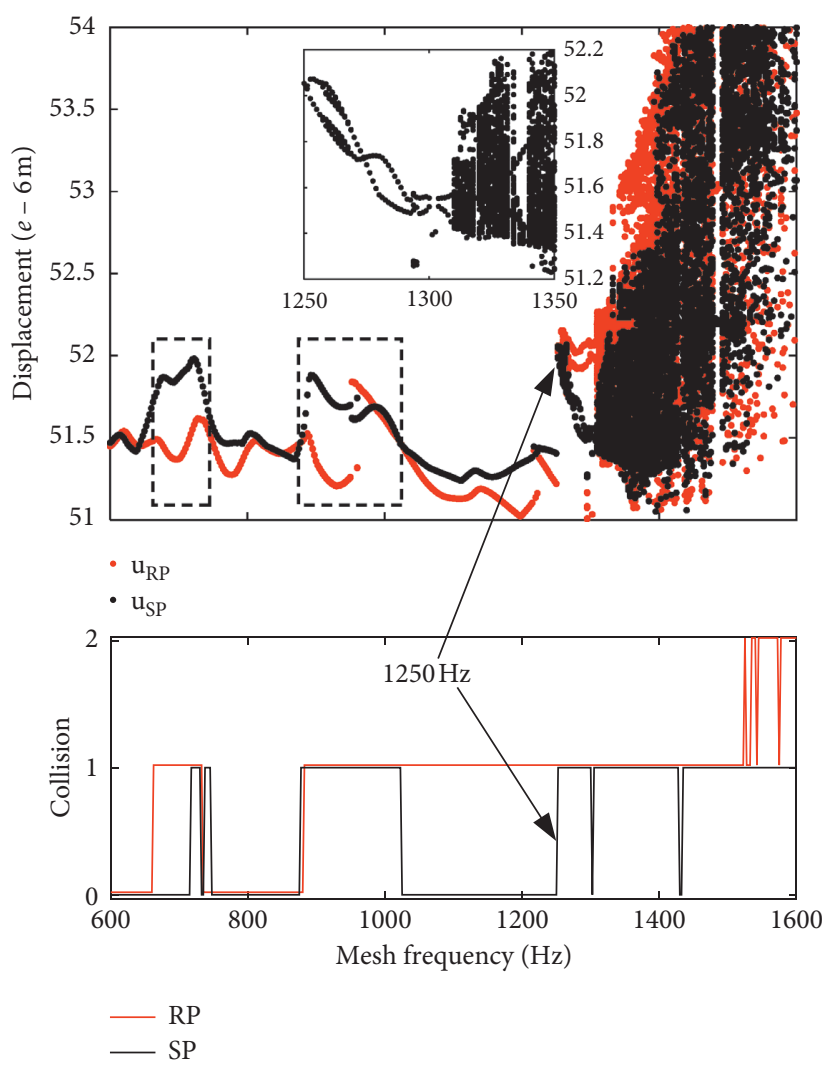

(a)
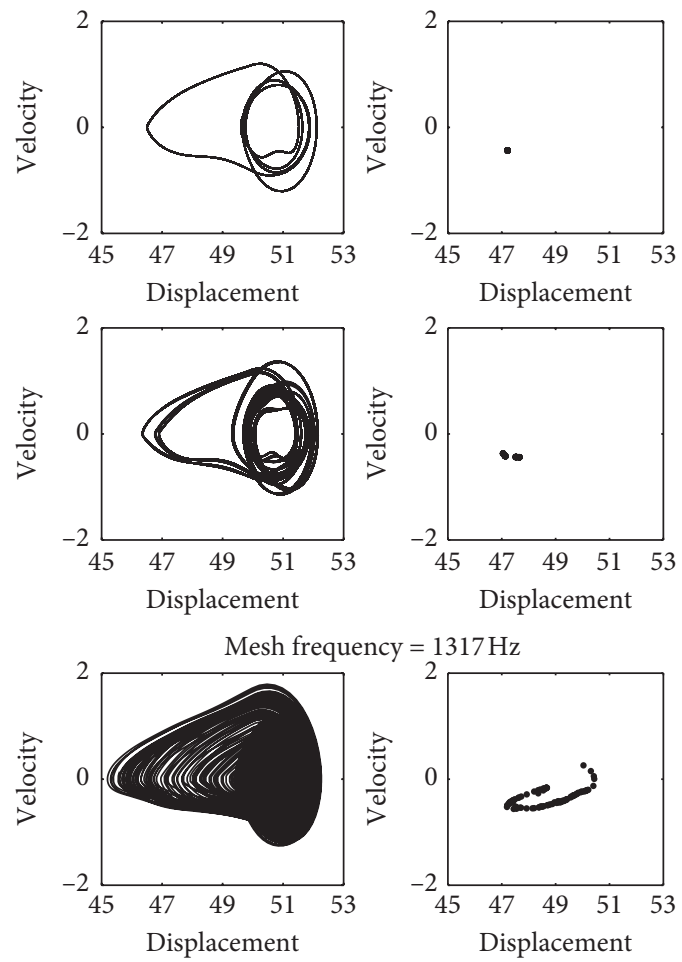

(b)

FIgURe 4: Nonlinear dynamics of the coupled system of the PGT and the SOWCs as rigid loads. (a) Bifurcation and collision diagrams of RP and SP. (b) Phase diagrams and Poincaré maps of RP on key mesh frequencies.

3.1.4. Collision Characteristics. A SOWC under one-way model can be in either a noncollision state or a collision state, whereas gears have three states known as noncollision, unilateral collision, and bilateral collision. Discriminants of each collision status are shown in Table 2.

3.2. Nonlinear Dynamics of the Unit. When $\omega_{C} \approx \omega_{S}$, the sun gear, the ring gear, and the carrier have almost the same speed and $f_{m}$ approaches to zero. Simulation results show that serious vibrations occur under this situation; therefore, homogenous speed of the sun gear and the carrier should be avoided and should be stepped over as fast as possible.

When $\omega_{S}>\omega_{C}$, the sun gear outputs power and functions on a relatively higher speed on positive direction; meanwhile, $\omega_{P i}<0$.

When $\omega_{S}<\omega_{C}$, the sun gear functions either on a relatively lower speed on positive direction (outputing power) or on any speed on negative direction (inputing power); meanwhile, $\omega_{P i}>0$. Nonlinear characteristics are similar for identical absolute value of $\omega_{P i}$ with different signs.

Case A is applied in this section. To work out influences of SOWCs, a rigid load model that reveals pure PGT dynamics by treating SOWCs as rigid loads is firstly portrayed in Figure 4. As shown in the collision diagram in Figure 4(a), $\mathrm{RP}$ has wider ranges of collisions, compared to SP, and falls into bilateral collisions after $1525 \mathrm{~Hz}$. Before $1250 \mathrm{~Hz}$, simultaneous collisions of SP and RP happen on ranges of $717.5-730 \mathrm{~Hz}$ and $882.5-1022.5 \mathrm{~Hz}$ and trigger several jump points; however, no serious vibration occurs. Collisions of SP and RP on $1250 \mathrm{~Hz}$ stimulate a jump on their bifurcation diagrams. Afterwards, period-doubling bifurcations of $1 \mathrm{~T}$ $2 \mathrm{~T}$ happen on range of $1251-1256 \mathrm{~Hz}$ and evolve into $4 \mathrm{~T}$ bifurcation from $1256 \mathrm{~Hz}$ to $1268 \mathrm{~Hz}$. The system returns to single period solution for a very small range of $1301 \mathrm{~Hz}$ to $1303 \mathrm{~Hz}$. After that, period-doubling bifurcations occur and evolve into chaos through quasiperiodic bifurcations. Phase diagrams and Poincaré maps of RP on $1250 \mathrm{~Hz}, 1260 \mathrm{~Hz}$, 

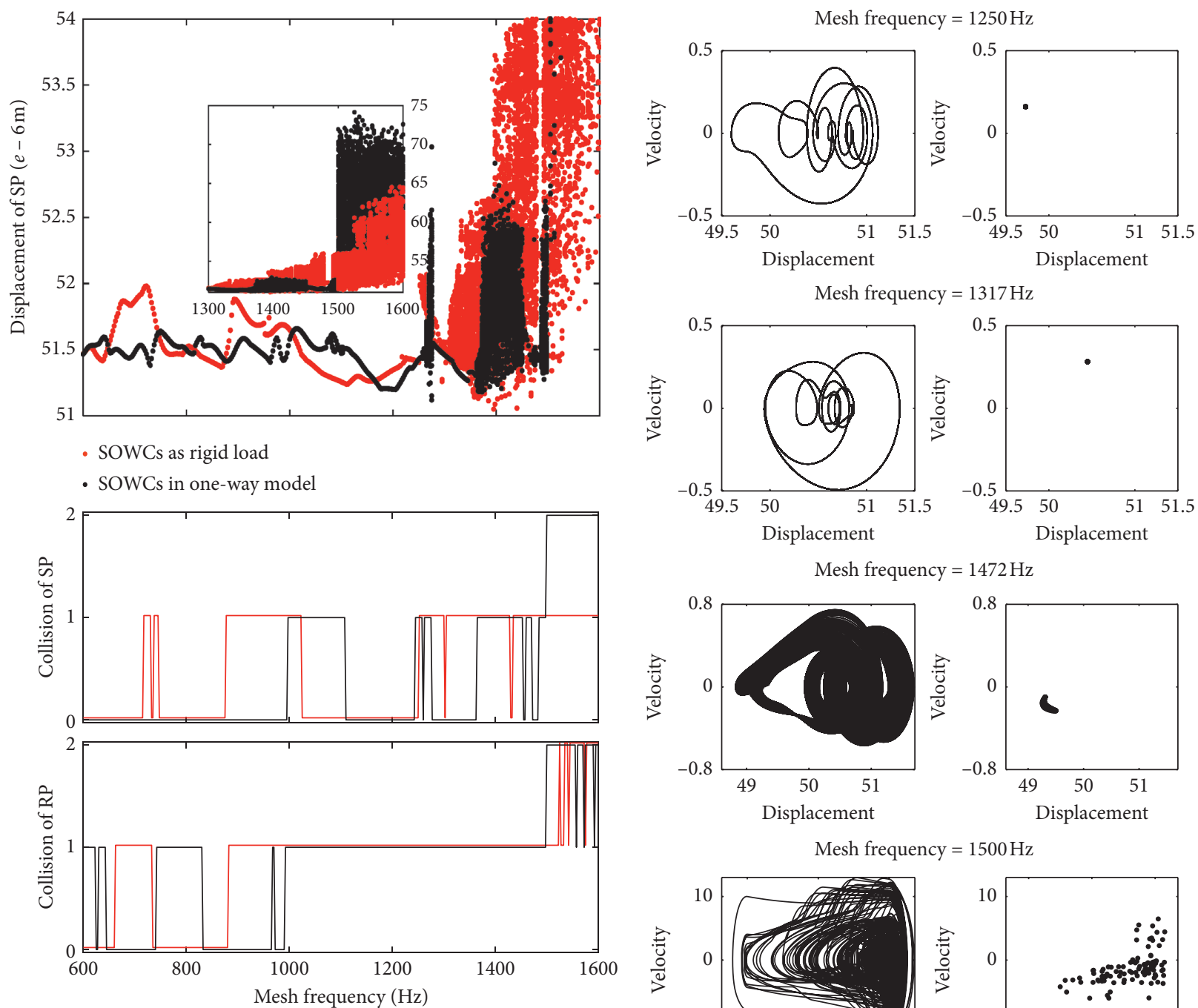

— SOWCs as rigid load
SOWCs in one-way model
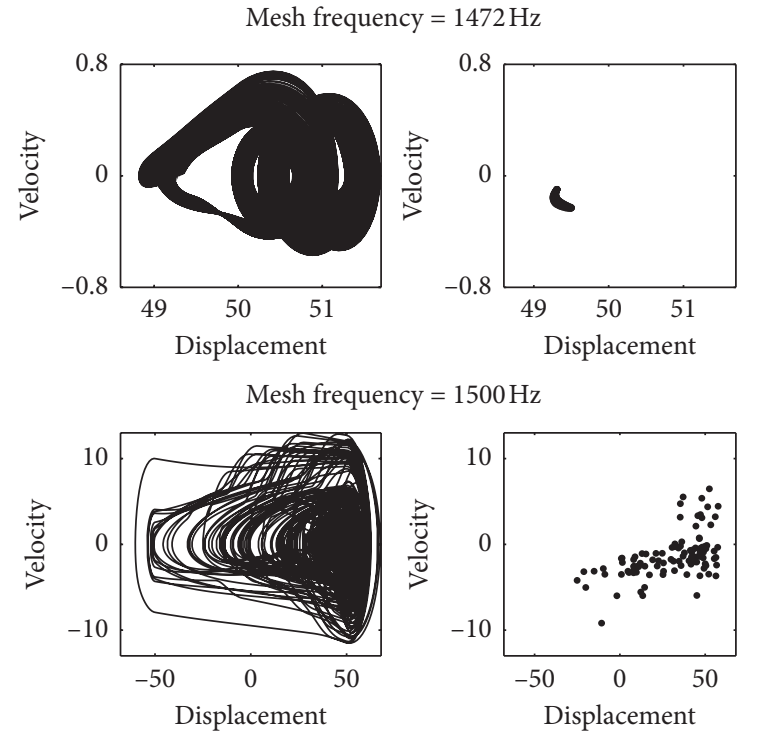

(a)

(b)

FIGURE 5: Nonlinear dynamics of the coupled system when SOWCs work as rigid loads or in one-way model. (a) Bifurcation and collision diagrams. (b) Phase diagrams and Poincaré maps of RP on key mesh frequencies when SOWCs work in one-way model.

and $1317 \mathrm{~Hz}$ are illustrated in Figure 4(b), revealing the 1T solution on the jump point, 4T solutions on the range of $1256 \mathrm{~Hz}$ to $1268 \mathrm{~Hz}$, and quasiperiodic solutions before the final chaos.

Figure 5 depicts the nonlinear dynamics of the PGT under the influences of SOWCs' one-way model. Collision comparisons of the one-way model and the rigid load model in Figure 5(a) show that the collision range in lower region of frequencies is narrowed; nevertheless, deteriorated bilateral collisions of PGT occurred when mesh frequency gets higher than $1500 \mathrm{~Hz}$. Bifurcation diagram shows that the jump does not happen for the one-way model at $1250 \mathrm{~Hz}$. Instead, the system quickly falls into chaos at the range of $1255-1277.5 \mathrm{~Hz}$ through $2 \mathrm{~T}, 4 \mathrm{~T}$, and quasiperiodic bifurcations. After $1277.5 \mathrm{~Hz}$, SP suddenly quits the collisions and returns to single period solutions until $1360 \mathrm{~Hz}$. During the range of $1360-1455 \mathrm{~Hz}$, the system experiences evolutions from single period solution to chaos and back to single period solution both through quasiperiodic bifurcations. From $1472 \mathrm{~Hz}$, the system falls into chaos rapidly through quasiperiodic bifurcations and evolves to bilateral collisions after $1500 \mathrm{~Hz}$.

Detailed differences can be observed by comparing phase diagrams and Poincaré maps of RP under the rigid model in Figure 4(b) and the one-way model in Figure 5(b). Comparisons of phase diagrams on $1250 \mathrm{~Hz}$ show that solution with one-way model of SOWCs is more convoluted; however, the collision is slightly alleviated based on the minimum displacement, which is larger than $49.5 e-6 \mathrm{~m}$ for the one-way model solution. Phase diagram on $1317 \mathrm{~Hz}$ shows 


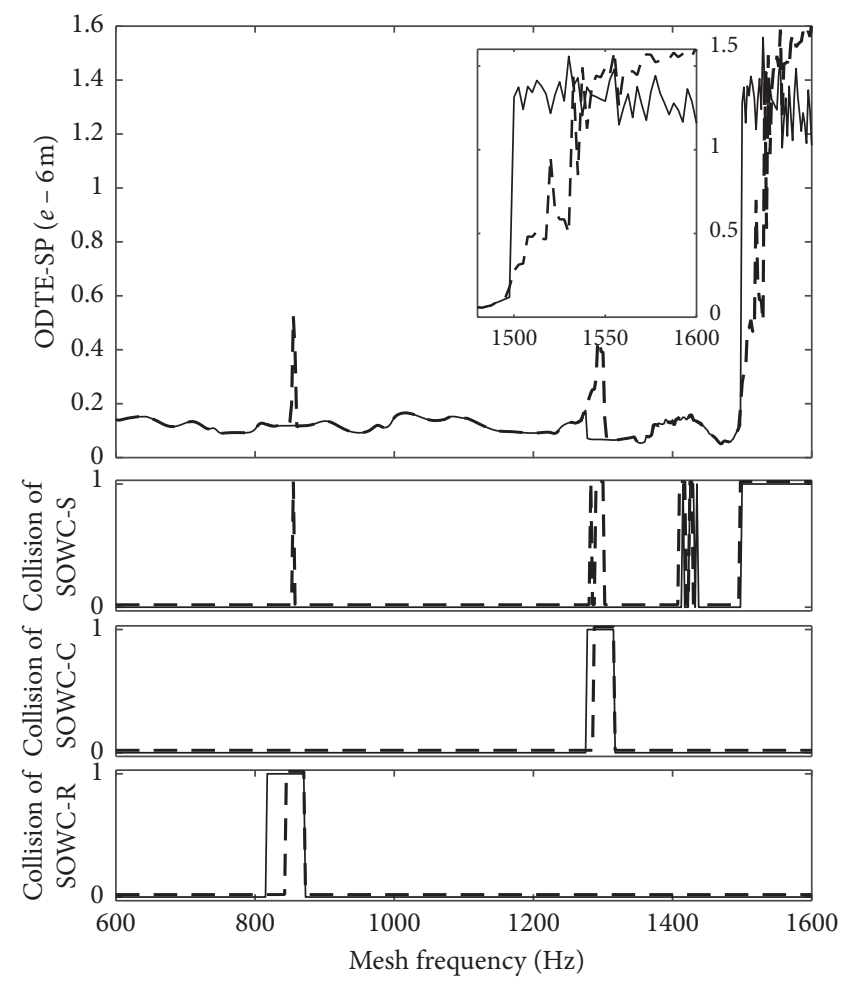

- - - Engaged model

- One-way model

(a)
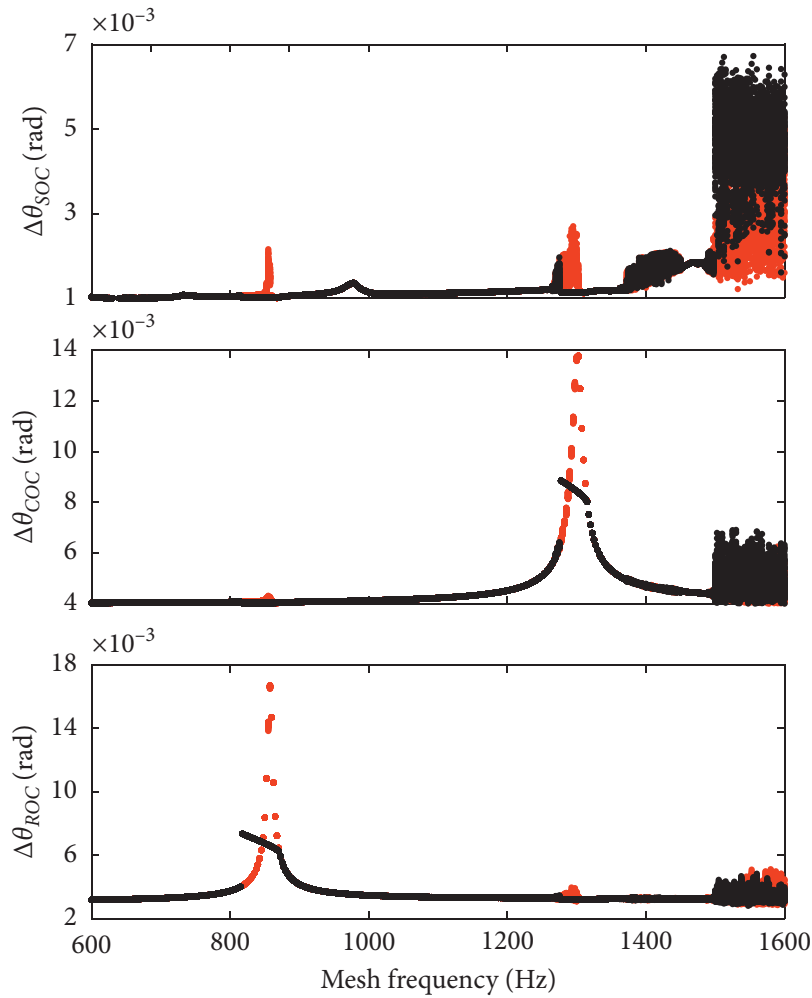

- Engaged model

- One-way model

(b)

FIGURE 6: Comparisons of the engaged model and the one-way model. (a) Vibration characteristics of SP and collision status of SOWCs. (b) Bifurcation diagrams of each SOWC.

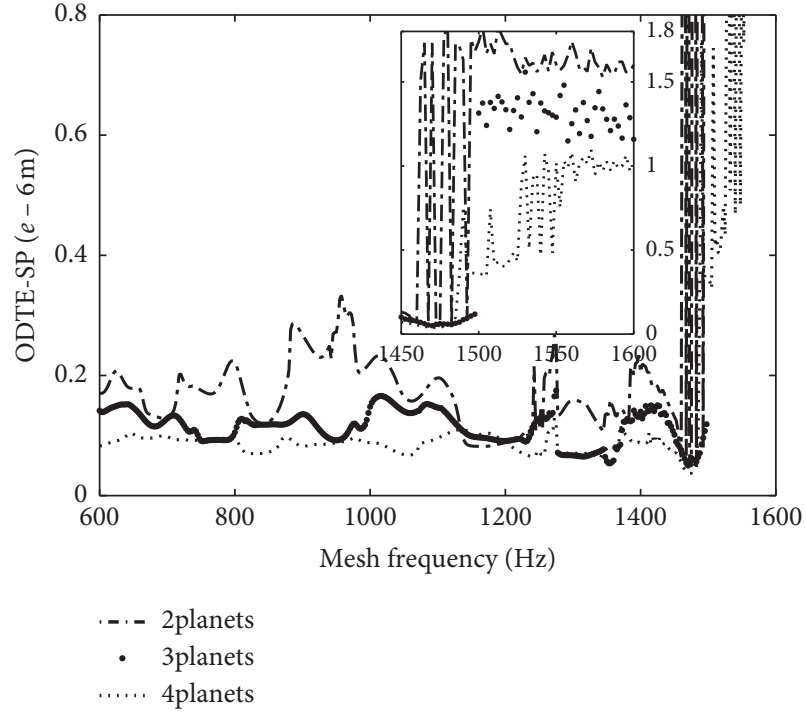

(a)

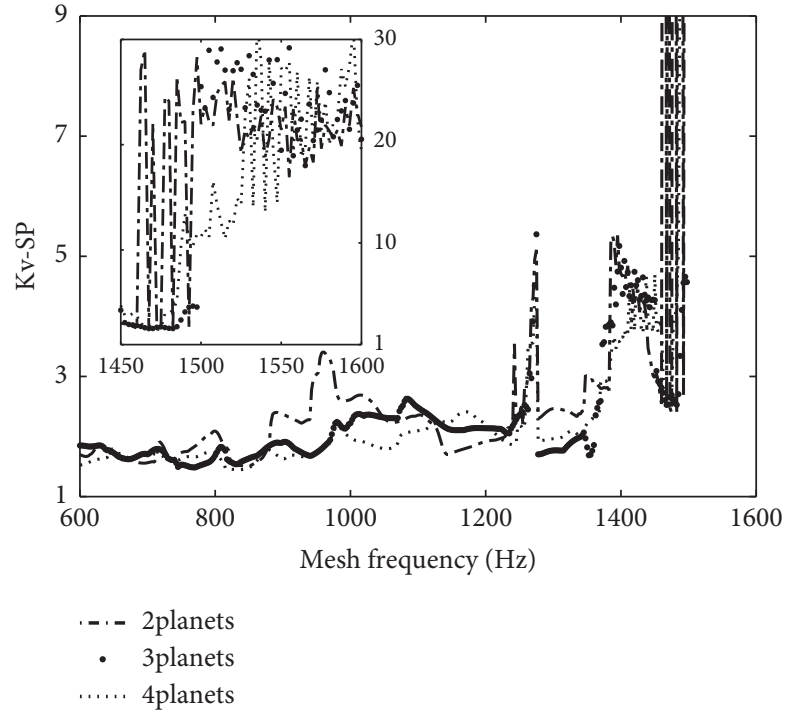

(b)

Figure 7: Influences of planet quantities. (a) Vibration characteristics of SP. (b) Load characteristics of SP. 

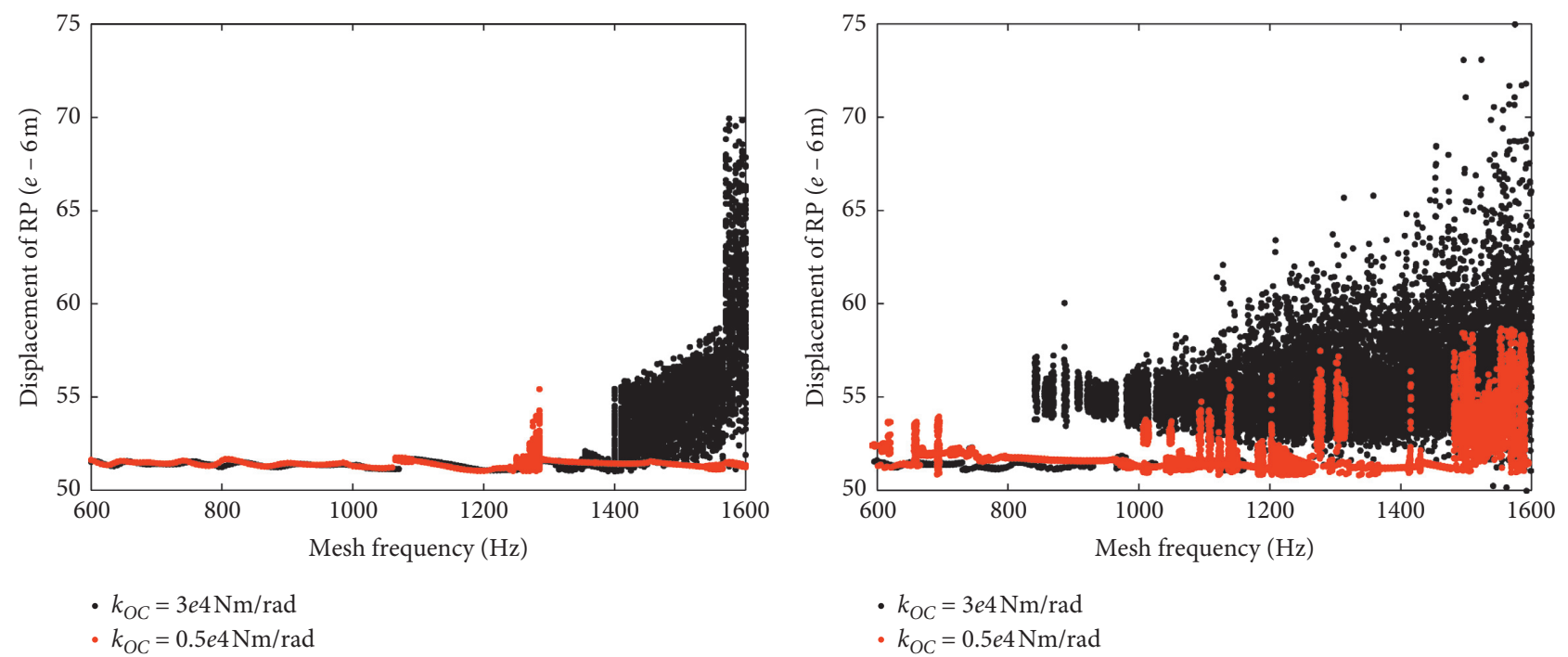

(a)

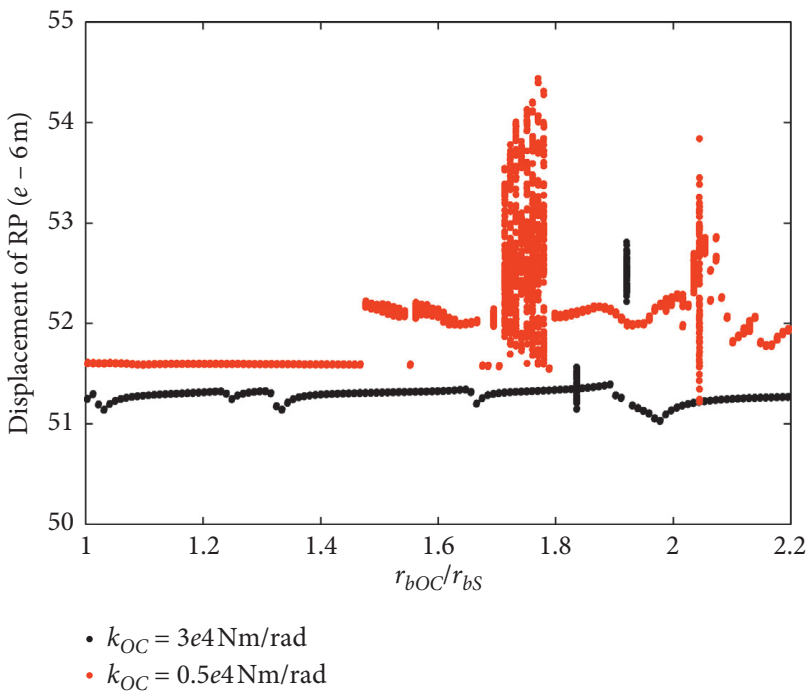

(c)

(b)

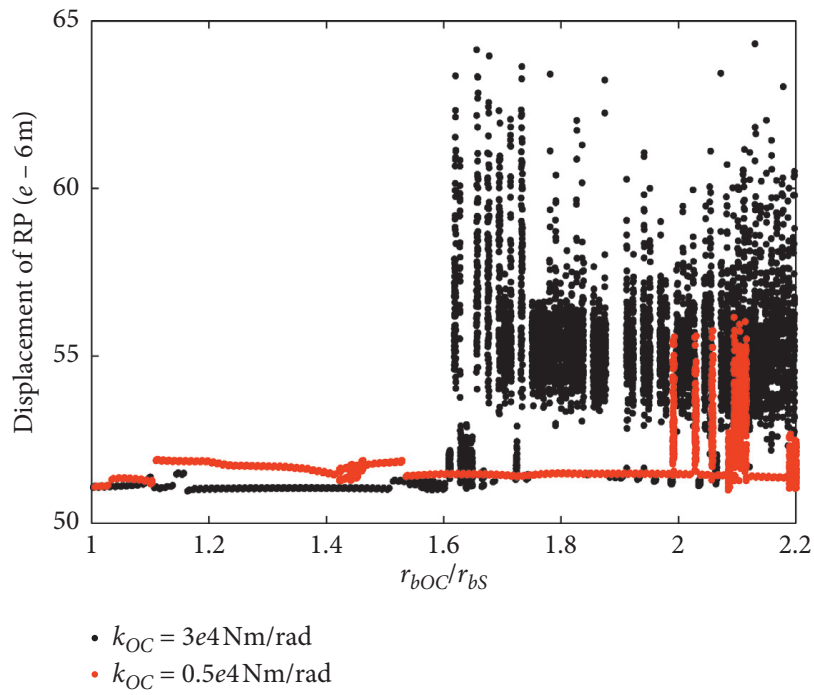

(d)

FIGURE 8: Stiffness and radius effects under Case B. (a) RP bifurcation diagrams when $r_{b O C}=r_{b s}$. (b) RP bifurcation diagrams when $r_{b O C}=r_{b C}$ and RP bifurcation diagrams with the changing radius at the mesh frequency (c) at $800 \mathrm{~Hz}$ and (d) at $1200 \mathrm{~Hz}$.

one solution on range $1277.5 \mathrm{~Hz}$ to $1360 \mathrm{~Hz}$. This range, standing for the sudden termination of previous range of chaos, overlaps with the point when SOWC-C begins to fall into collision (introduced in the next paragraph). Phase diagram on $1472 \mathrm{~Hz}$ and $1500 \mathrm{~Hz}$ illustrates the quasiperiodic solutions and bilateral collision solutions of the system, respectively.

To further work out the influences associated with SOWCs, an engaged model is compared with the one-way model in Figure 6. The engaged model stands for the idealized two-way transmission by neglecting the idle angle. In other words, the engaged model assumes that direction switches during transmissions of the SOWCs have no interruptions. Figure 6(a) illustrates the vibration characteristics of SP and collision status of each SOWCs under both models. The engaged model can delay the bilateral collisions of PGT on $1500 \mathrm{~Hz}$; however, the price is the exaggerated vibration during the bilateral collisions. Besides, series vibrations occur under engaged model on ranges of $852.5-862.5 \mathrm{~Hz}$ and $1277.5-1305 \mathrm{~Hz}$, associated with the shorter range of collisions on SOWC-C and SOWC-R.

Bifurcation diagrams of SOWCs are illustrated in Figure 6(b). Bifurcation diagrams of SOWC-C and SOWC-R show the jumping phenomenon of SOWCs and reveal similar responses on belt-drive dynamic systems with a oneway clutch $[13,14]$. Additionally, resonance responses of PGT on range $1277.5-1318 \mathrm{~Hz}$ of one-way model disappear in accordance with the jump point and collision of SOWC-C on range $1277.5-1318 \mathrm{~Hz}$. Similar responses happen to SOWC-R. The resonance area moves right side as dimensionless stiffness $k_{O C} / m_{O C} r_{O C}^{2}$ increases. It is worth noticing that responses of SOWC connected to the sun gear are always more in accordance with the PGT. 


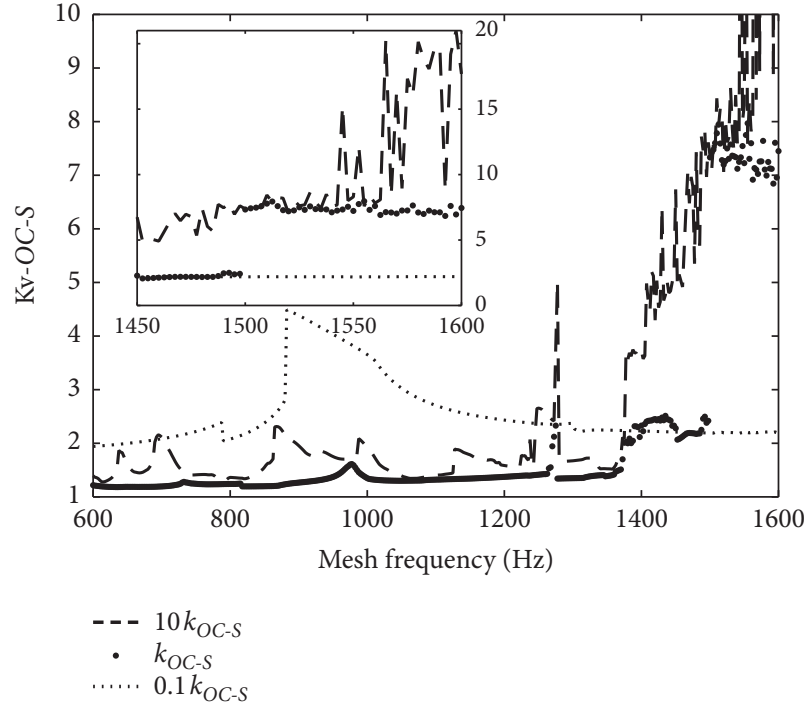

(a)
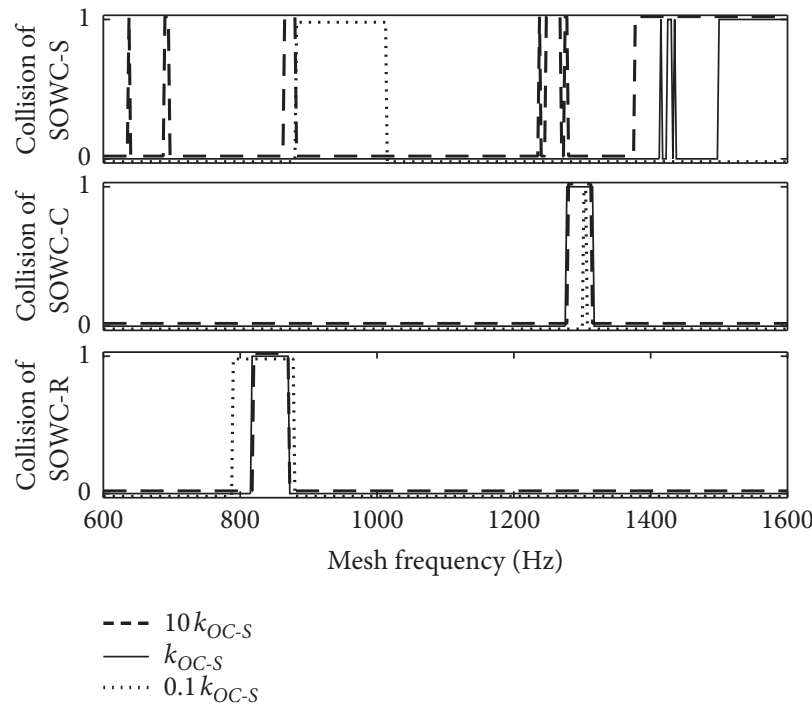

(c)

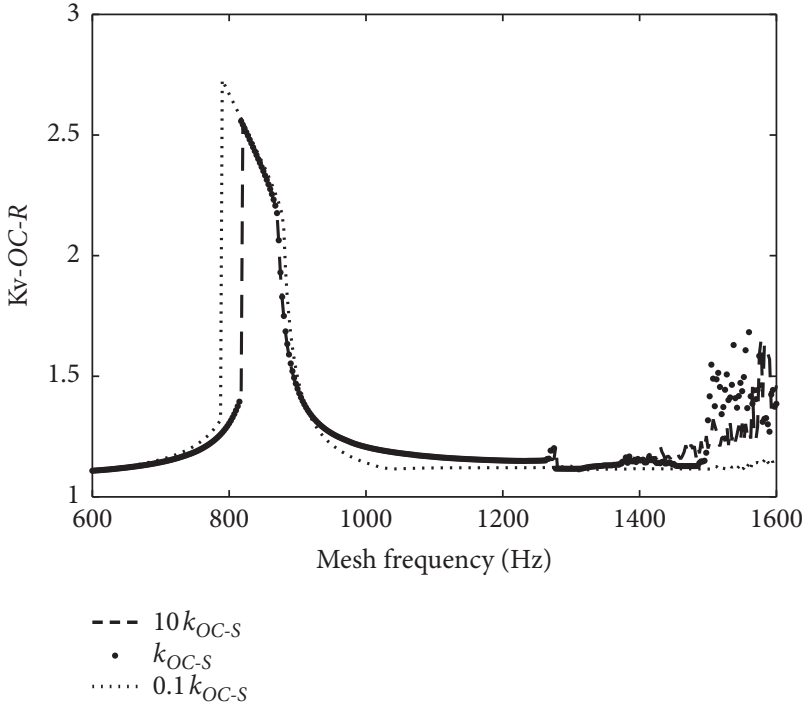

(b)

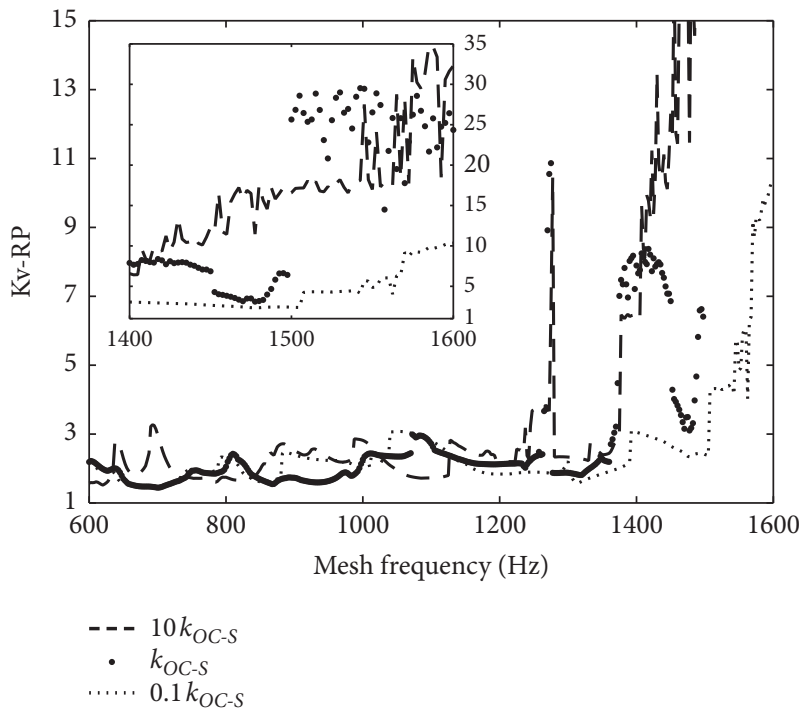

(d)

FIGURE 9: Nonlinear dynamics of the unit as stiffness of SOWC-S changed. (a) Load characteristics of SOWC-S. (b) Load characteristics of SOWC-R. (c) Collision of each SOWC. (d) Load characteristics of RP.

Influences of the planet quantities in the PGT are revealed in Figure 7. PGTs with two planetary gears, three planetary gears, and four planetary gears are compared. Planet quantity's influences to the overall bifurcation structure of the PGT are limited; however, vibrations and maximum dynamic loads decrease with the increasing number of the planet gear. Besides, the range of severe vibrations can also be diminished with more planets.

\section{Parameter Configurations of SOWCs}

Attenuation of torsional oscillations is important in automotive powertrains. Parameters of SOWCs can also have great effects on the transmission quality. The influences of SOWCs' radius and stiffness are firstly investigated using three identical SOWCs. To further investigate parameter influences of each SOWC, 0.1 times, 10 times of the nominal stiffness and 10 times, 50 times of the nominal damping ratio are applied under case A.

4.1. Overall Influences of Radius and Stiffness. The stiffness for a roller type SOWC changes with material, size, and other factors. This paper focuses on the stiffness range from $0.5 e 4$ to $3 e 4 \mathrm{Nm} / \mathrm{rad}$. On the other hand, simulation results show that radius of SOWCs can also have great effects on the dynamic of the transmission unit. bifurcation structures of different stiffness and radius of all the SOWCs under Case B are firstly explored in Figure $8(\mathrm{a})$ and $8(\mathrm{~b})$ Bifurcation characteristics with the changing radius of different stiffness on different frequencies are studied in Figures $8(\mathrm{c})$ and $8(\mathrm{~d})$. 


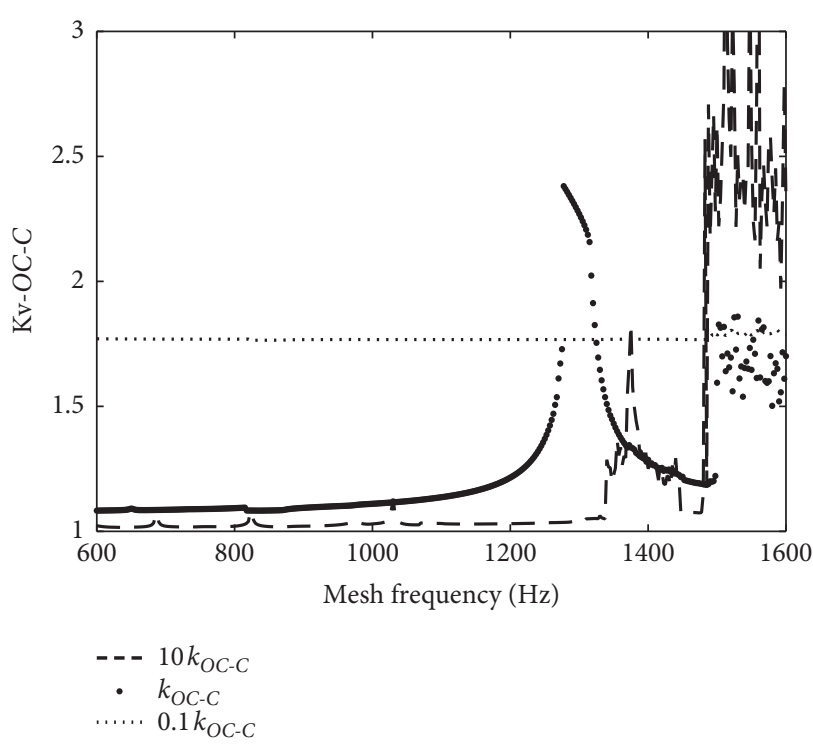

(a)

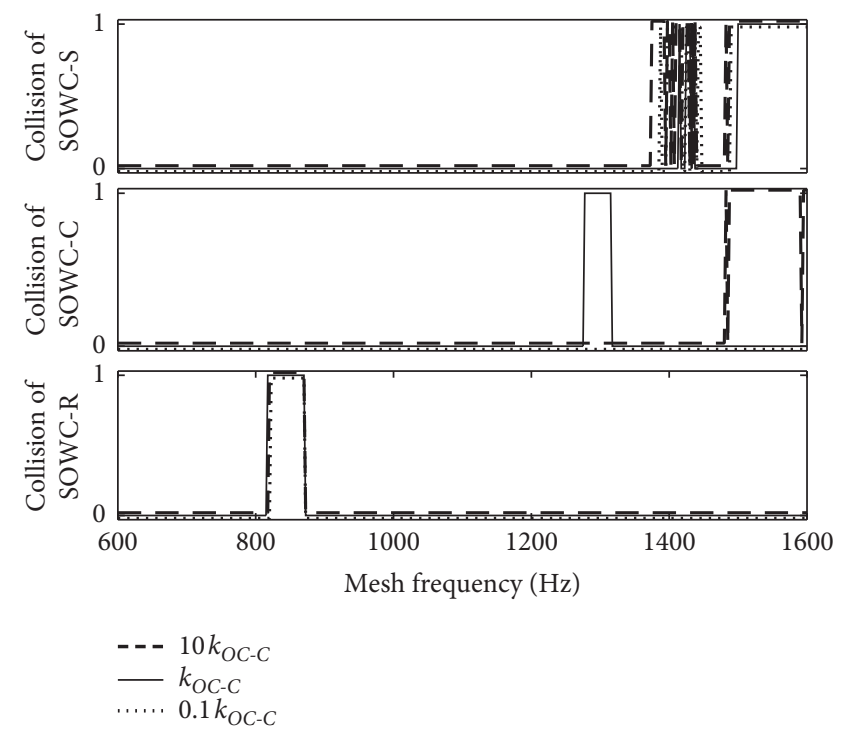

(c)

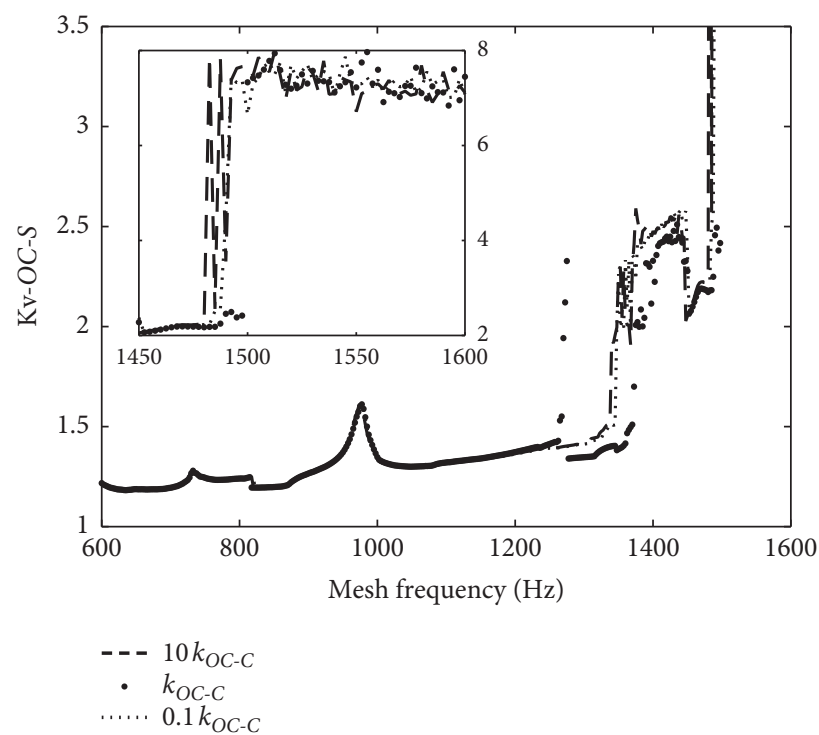

(b)

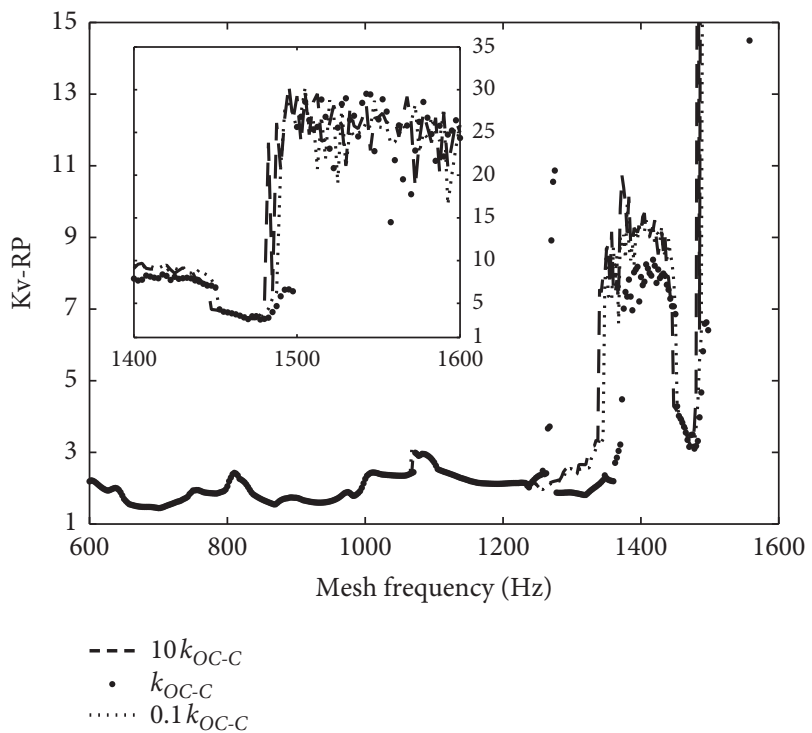

(d)

FIGURE 10: Nonlinear dynamics of the unit as stiffness of SOWC-C changed. (a) Load characteristics of SOWC-C. (b) Load characteristics of SOWC-S. (c) Collision of each SOWC. (d) Load characteristics of RP.

As shown in Figure 8(a), where $r_{b S}$ is applied to each SOWC, increase of the stiffness of SWOCs has little influence to the bifurcation structure to RP on frequencies below $1200 \mathrm{~Hz}$. After $1200 \mathrm{~Hz}$, the resonances on range $1260-1285 \mathrm{~Hz}$ for $k_{O C}=0.5 e 4 \mathrm{Nm} / \mathrm{rad}$ do not apply to responses when $k_{O C}=3 e 4 \mathrm{Nm} / \mathrm{rad}$. However, the increase of $k_{O C}$ renders the PGT fall into chaos after $1400 \mathrm{~Hz}$ and intensify into bilateral collisions after $1570 \mathrm{~Hz}$. Similar qualitative conclusions can also be drawn from Figure 8(b) that the increasing of the stiffness of SOWCs render the PGT fall into chaos on lower range of frequencies and aggregate the vibrations. Comparisons from Figure 8(a) and 8(b) show that the increase of radius of SOWCs renders discontinuous quasiperiodic or chaos solutions on lower ranges of frequency and intensifies vibrations with wider unstable solution scopes and severe collisions on high frequency ranges. This trend gets more distinct when $k_{O C}$ is increased from $0.5 e 4 \mathrm{Nm} / \mathrm{rad}$ to $3 e 4 \mathrm{Nm} / \mathrm{rad}$.

Bifurcation diagrams with the changing radius on $800 \mathrm{~Hz}$ in Figure 8(c) show that on relatively lower frequency, the increase of radius of SOWCs has limited effects to the PGT, even for the resonance oscillations within the range of 1.6 to 1.8 times of $r_{b s}$. This situation changes when the mesh frequency turns higher. As shown in Figure 8(d) on $1200 \mathrm{~Hz}$, for the system with harder SOWCs, RP falls into chaos and severe vibrations after 1.6 times of $r_{b s}$ through quasiperiodic bifurcations. Though the PGT seems to be insensitive with the increasing radius of SOWCs when 


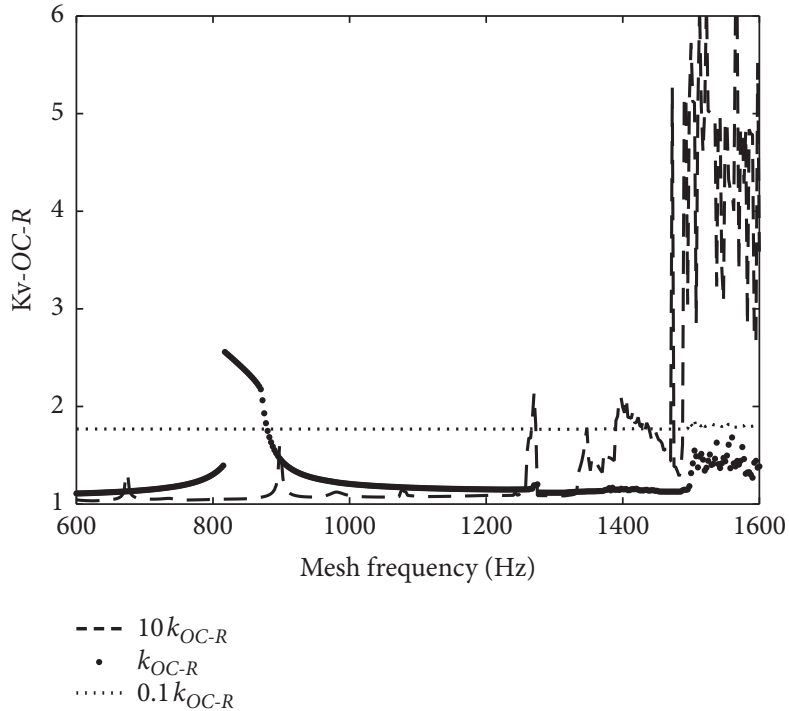

(a)

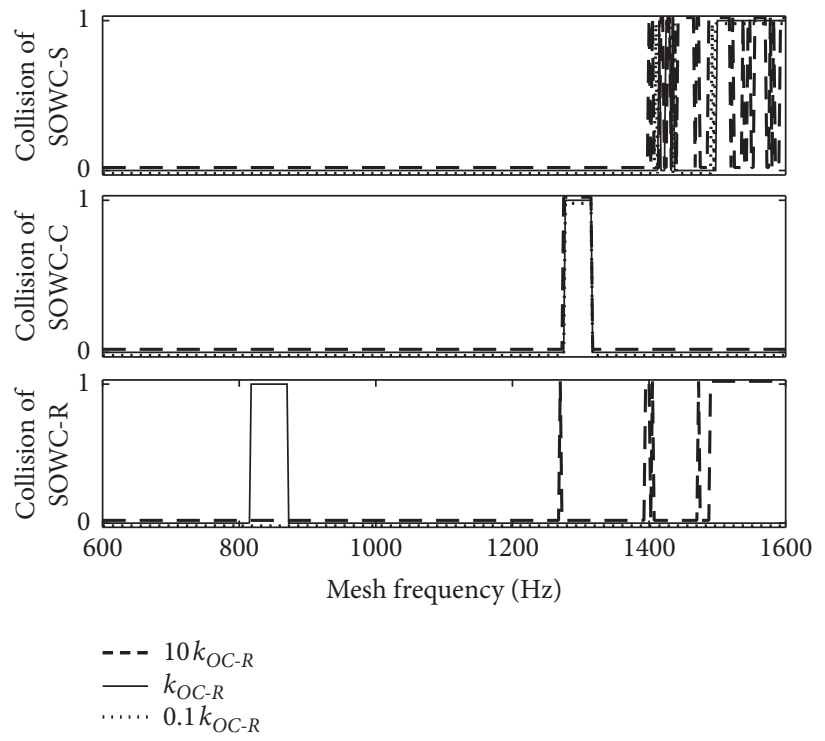

(c)

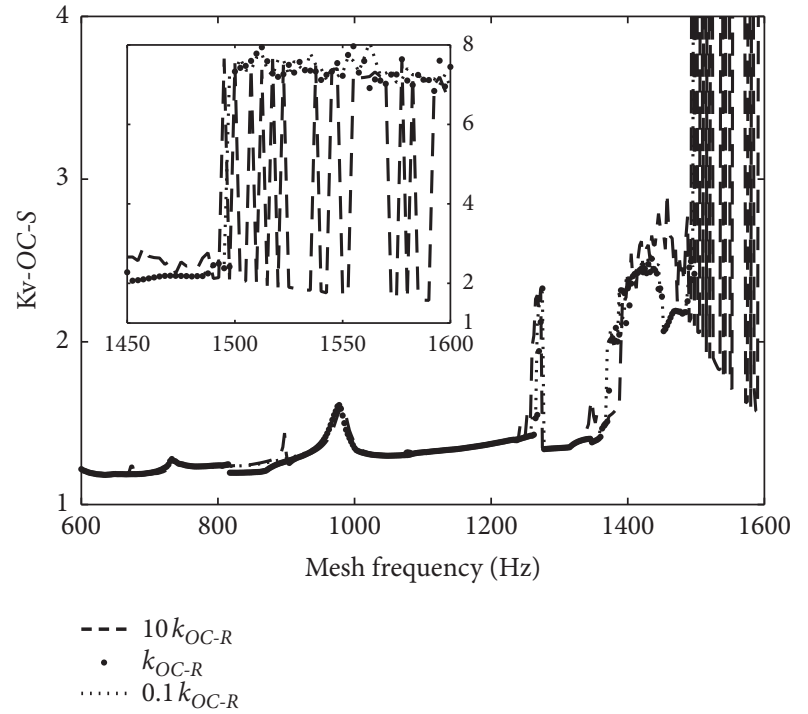

(b)

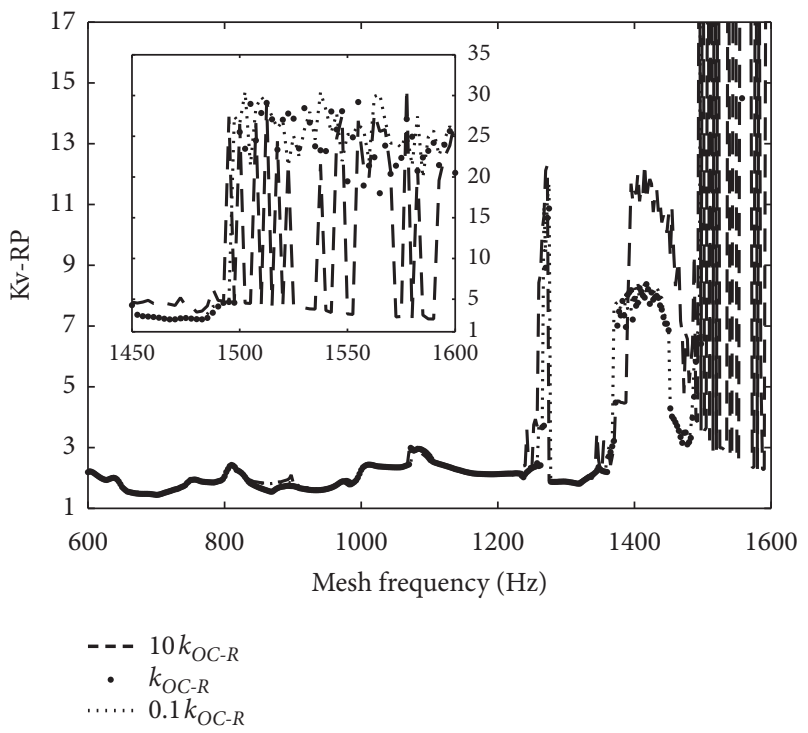

(d)

FIGURE 11: Nonlinear dynamics of the unit as stiffness of SOWC-R changed. (a) Load characteristics of SOWC-R. (b) Load characteristics of SOWC-S. (c) Collision of each SOWC. (d) Load characteristics of RP.

stiffness of SOWCs is low, vibration and load characteristics of SOWCs get deteriorated. Furthermore, detailed comparisons among Figures 5(a), 8(a), and 8(b) reveal that of the three SOWCs, radius of SOWC-S acts as the main factor to the overall structure of the PGT bifurcations.

4.2. Stiffness Effects of Each SOWC. Stiffness effects of the SOWC-S are illustrated in Figure 9. As shown in Figure 9(d), the effects to the PGT seem to be convoluted on lower frequency range below $1200 \mathrm{~Hz}$; nevertheless, it is straightforward on higher frequency range above $1200 \mathrm{~Hz}$ that the increase of $k_{O C-S}$ deteriorated load characteristics of the PGT. Dynamic loads get larger, and resonance responses get wider for the resonance responses of PGT on range
$1277.5-1318 \mathrm{~Hz}$ of the original system. The PGT also gets easier to fall into chaos with larger $k_{O C-S}$ on meshing frequencies higher than $1400 \mathrm{~Hz}$. In addition, a wider range of oscillations is stimulated for SOWC-S because of the deteriorated vibrations as shown in Figure 9(c).

On the range below $1200 \mathrm{~Hz}$, maximum dynamic load of SOWC-S increases and they are reflected on the PGT. However, the influences can hardly be observed on SOWC-R (shown in Figure 9(b)) or SOWC-C (reflected in Figure 9(c) through the collision of SOWC-C). . When SOWC-S gets too soft, the decrease of $k_{O C-S}$ enlarges the DTE and renders larger dynamic loads and series vibrations to itself. Resonances of SOWC-R get intensified because of the direct transmission of DTE through planetary gears. Howbeit, resonances of SOWC-C are weakened. 


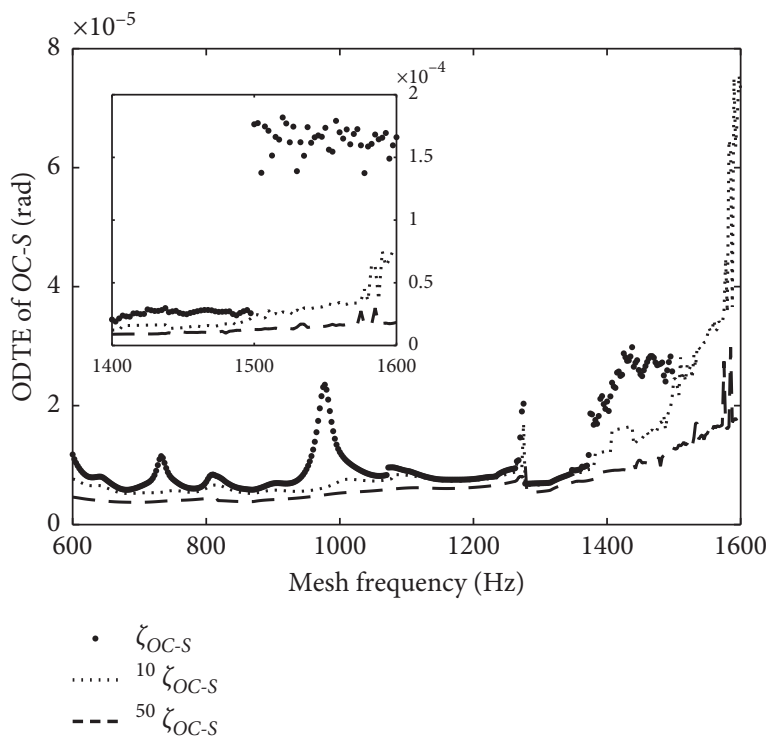

(a)

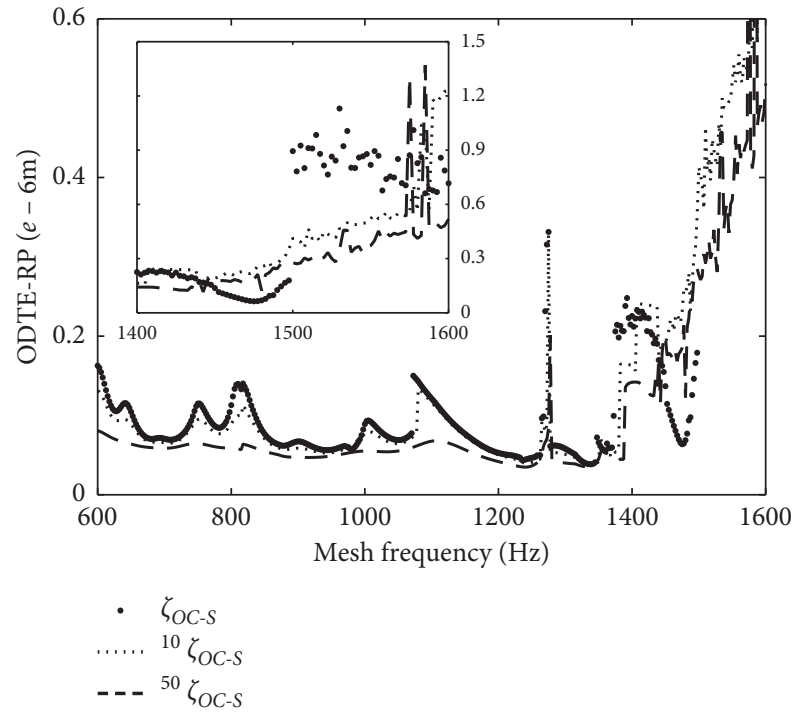

(c)

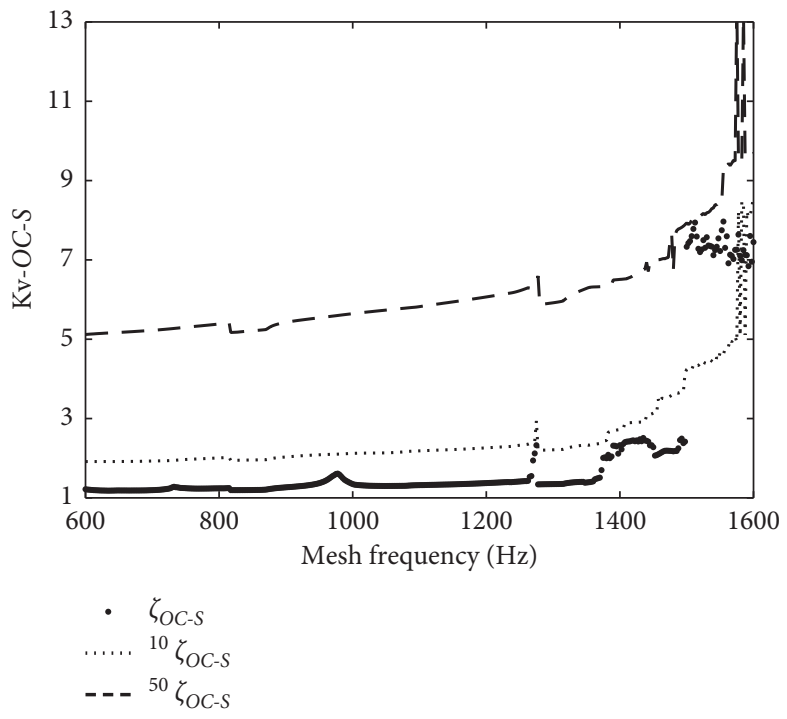

(b)

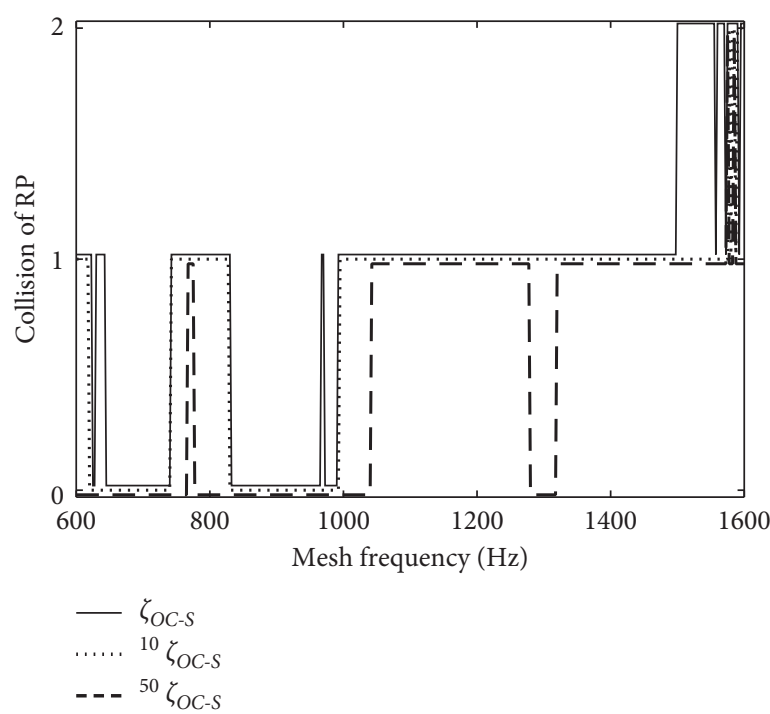

(d)

Figure 12: Nonlinear dynamics of the unit as damping ratio of SOWC-S changed. (a) Vibration characteristics of SOWC-S. (b) Load characteristics of SOWC-S. (c) Vibration characteristics of RP. (d) Collision of RP.

Stiffness effects of the SOWC-C are illustrated in Figure 10. Different from stiffness influences of SOWC-S to itself illustrated in Figure 9(a), the increased stiffness of SOWC-C decreases the dynamic loads of itself below 1277.5 Hz, as shown in Figure 10(a). On the same range, load characteristics of SOWC-C deteriorate when SOWC-C gets too soft. However, none of these situations evidently influences the dynamics of the PGT, the SOWC-S, or the SOWC-R on the range (the changing of resonance region with different stiffness shall be excluded).

On the chaos ranges of the PGT, despite the stiffness influences to the resonance frequency, increase of $k_{O C-C}$ intensifies the vibration of SOWC-C, but mitigates the vibration of the PGT. This situation is in accordance with the collision of SOWC-C as shown in Figure 10(c).

Stiffness effects of the SOWC-R are illustrated in Figure 11. When SOWC-R gets too soft, load characteristics of SOWC-R are deteriorated. The increased stiffness of SOWC-R decreases the dynamic loads of itself on nonchaos regions of the PGT. This trend, the influence of the changed object to the dynamics of itself, are accordant with the effcets of SOWC-C, but opposite to the effects of SOWC-S. Stiffness effects of SOWC-R to the PGT on the steady region are similar with the effects of SOWC-C that the PGT are seldomly affected. However, the influences on the chaos region are different. 


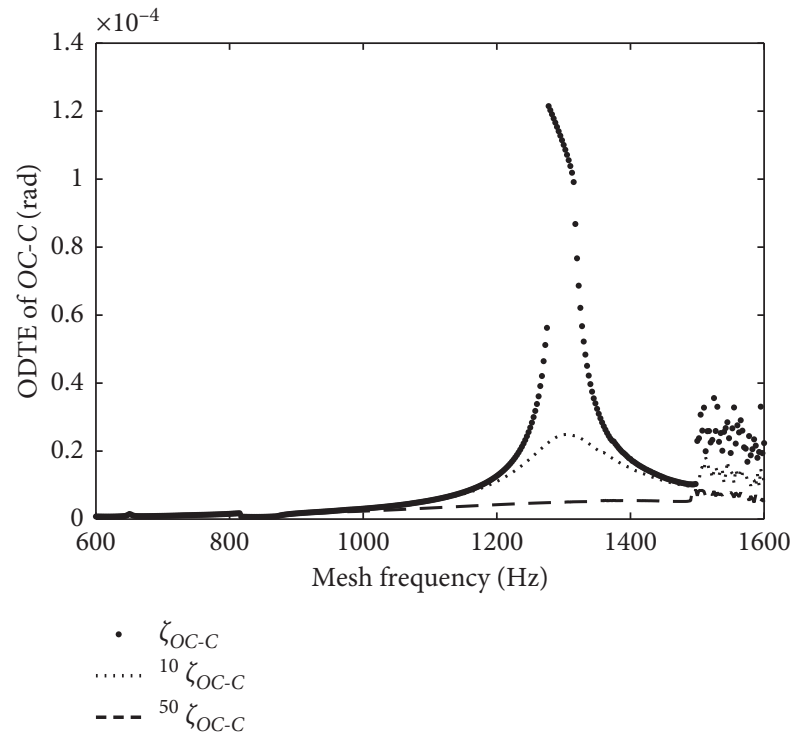

(a)

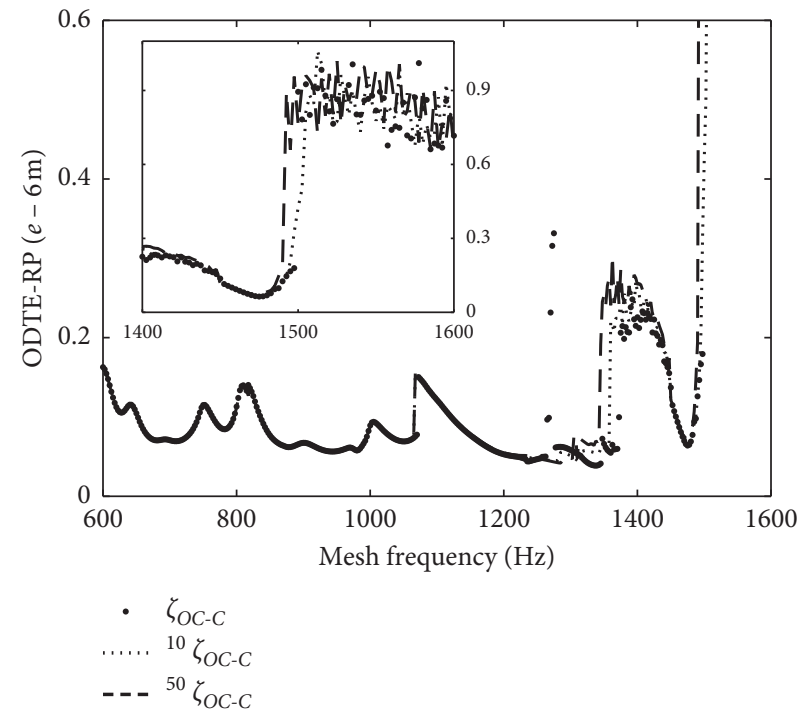

(b)

FIGURE 13: Nonlinear dynamics of the unit as damping ratio of SOWC-C changed: vibration characteristics of (a) SOWC-C and (b) RP.

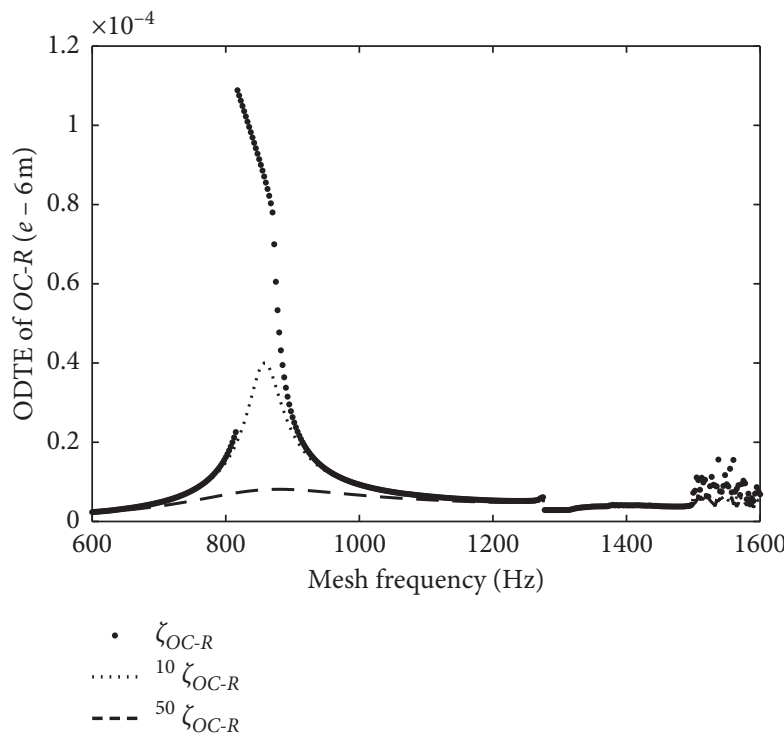

(a)

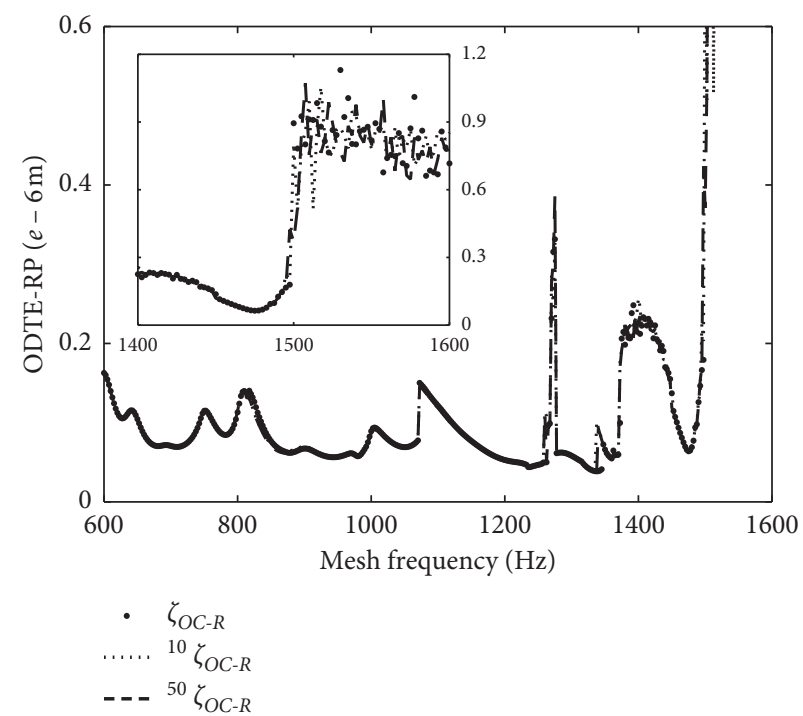

(b)

FIGURE 14: Nonlinear dynamics of the unit as damping ratio of SOWC-R changed: vibration characteristics of (a) SOWC-R and (b) RP.

As shown in Figures 11(a) and 11(d), on the range of $1255-1277.5 \mathrm{~Hz}$ where chaos occurs to the PGT, the augment of stiffness of SOWC-R enlarges the dynamic loads of the PGT and SOWC-R. Similar responses happen on the range of $1332.5-1482.5 \mathrm{~Hz}$. On the higher frequency region where the PGT involves into serious bilateral collisions, the augment of stiffness of SOWC-R inconsecutively decrease the vibration of the PGT because of the transitory interruption of the torque transmission (as shown in the collision diagram of SOWC-R in Figure 11(c)). Nevertheless, the solutions are unstable within the range, and load characteristics of SOWC-R are enormously exasperated.
4.3. Damping Ratio Effects of Each SOWC. Damping ratio effects of the SOWC-S are shown in Figure 12. As shown in Figure 12(a), the increase of $\zeta_{O C-S}$ decreases the DTE and vibration characteristics of SOWC-S and hence the possibility of its collision. The diminishments are remarkable especially near resonance points or during chaos. However, the load characteristics of SOWC-S are enlarged integrally as shown in Figure 12(b). Similar load characteristics work to SOWC-C and SOWC-R when their damping ratios are increased. Effects of $\zeta_{O C-S}$ on the PGT are illustrated in Figure 12(c). As mentioned previously that responses of SOWC connected to the sun gear are always more in 
accordance with the PGT, consequently, the increase of $\zeta_{\text {OC-S }}$ diminishes the ODTE of the PGT within the entire range. Collisions of the PGT are also alleviated with larger $\zeta_{O C-S}$ as shown in Figure 12(d).

Damping ratio effects of the SOWC-C are shown in Figure 13. Different from the responses of SOWC-S, the increase of $\zeta_{\text {OC-C }}$ only diminishes the vibrations near resonance point and their effects to the PGT are limited. As shown in Figure 13(b), the chaos on the range of $1255-1277.5 \mathrm{~Hz}$ disappears because of the diminishment effects of larger damping ratio as shown in Figure 13(a).

Damping ratio effects of SOWC-R are shown in Figure 14. The damping ratio effects of SOWC-R are similar with that of SOWC-C that influences can only be observed near the resonance point. Effects of the increasing $\zeta_{O C-R}$ can hardly be observed as shown in Figure 14(b).

\section{Conclusions}

A nonlinear torsional model of a single-stage PGT coupled with three SOWCs is proposed. Piecewise nonlinearities of SOWCs associated with gear clearance, time-varying mesh stiffness, and synthetic transmission error are considered in the model. Different from most of the research studies on PGT that have one element fixed, the proposed PGT contains two DOF to reflect the power-split working condition of a hybrid transmission. Based on the specified group of comprehensive evaluation indices, nonlinear dynamics of the system and the influences of piecewise nonlinearities of the SOWCs are studied. Parameter influences of each of the three SOWCs are investigated. The main conclusions are as follows:

(1) One-way model of the SOWCs aggravates bilateral vibrations on high frequency range, but it also contributes to diminish the collision range of the PGT and oscillations. Additionally, transitory interruption of transmission of the one-way model contributes to reduce resonances and vibrations of bilateral collisions of the PGT.

(2) Influences of the planet quantity to the overall bifurcation structure of the unit are limited; however, vibration range and intensity decrease with the increasing number of the planet gear.

(3) Stiffness and radius of SOWCs greatly affect the bifurcation structure of the PGT. Increase of stiffness and radius renders series vibrations especially on higher frequencies. Of the three SOWCs, radius effects of the SOWC connected to the sun gear of the PGT dominate. Besides, for all three SOWCs, load characteristics of the SOWC deteriorate when the SOWC gets too soft.

(4) The increase of stiffness of the SOWC connected to the sun gear of the PGT deteriorates load characteristics of the SOWC and the PGT and renders the PGT fall into chaos on lower frequencies.

(5) The increase of stiffness of the SOWC connected to the carrier of the PGT mitigates the maximum dynamic loads of itself on low frequency range; however, the influences to the PGT are limited. On high frequency range, although the collisions of the SOWC stimulated by the larger stiffness decrease the maximum dynamic loads of the PGT, the influences are weak.

(6) The influences of increased stiffness of the SOWC connected to the ring gear of the PGT are similar with the influences connected to the carrier. Although the transitory interruption of transmission caused by collisions of the SOWC decreases the vibration of the PGT obviously, the solutions are unstable within the range, and load characteristics of the SOWC are enormously exasperated.

(7) Increase of the damping ratio of the SOWC connected to sun gear can distinctly reduce the vibration and maximum load of the PGT on the entire working range.

\section{Data Availability}

The data used to support the findings of this study are available from the corresponding author upon request.

\section{Conflicts of Interest}

The authors declare that they have no conflicts of interest.

\section{Acknowledgments}

This study was supported by the National Natural Science Foundation of China (grant no. 61074104). The authors are grateful to Prof. Tian Tian, Dr. Chen Chen, and Dr. Peng Wang for the revising advices and to Prof. Jintao Cheng for English writing suggestions.

\section{References}

[1] A. D. Gatta, L. Iannelli, M. Pisaturo, A. Senatore, and F. Vasca, "A survey on modeling and engagement control for automotive dry clutch," Mechatronics, vol. 55, pp. 63-75, 2018.

[2] D. Grzelczyk and J. Awrejcewicz, "Wear processes in a mechanical friction clutch: theoretical, numerical, and experimental studies," Mathematical Problems in Engineering, vol. 2015, Article ID 725685, 28 pages, 2015.

[3] I. Minas, N. Morris, S. Theodossiades, and M. O'Mahony, "Influence of clutch tribodynamics on drivetrain noise, vibration and harshness, (NVH), phenomena," in Proceedings of the SAE Technical Paper Series, SAE International, Warrendale, PA, USA, June 2018.

[4] S. H. Swales and A. J. Corsetti, "Selectable one-way clutch," US Patent US9181993B1, 2015.

[5] N. J. Bird, "Magnetically actuated clutch assembly," US Patent US9366298B2, 2016.

[6] H. Shioiri, H. Komada, H. Shibata, Y. Kurosaki, and H. Yasui, "Fixation structure for selectable one-way clutch," US Patent US9841068B2, 2017.

[7] A. C. Cioc, D. F. Greene, D. V. Dorigo, and S. A. Steele, "A selectable one-way clutch apparatus," US Patent US20170138414A1, 2017. 
[8] B. Schoolcraft, "Electromagnetically-actuated directionsensing roller clutch," US Patent US9856929B2, 2018.

[9] J. J. Prout, B. A. Pawley, and T. O. Hendrick, "Coupling and control assembly," US Patent US9188170B2, 2015.

[10] F. Samie, C. J. Lee, and B. Pawley, "Selectable one-way clutch in GM's RWD 6-speed automatic transmissions," SAE International Journal of Engines, vol. 2, no. 1, pp. 307-313, 2009.

[11] N. J. Bird, R. Bindra, and J. Klaser, "Development and challenges of electrically selectable one-way clutches," $S A E$ International Journal of Engines, vol. 10, no. 3, pp. 1338-1350, 2017.

[12] K. Zhao, Y. Liu, X. Huang, R. Yang, and J. Wei, "Uninterrupted shift transmission and its shift characteristics," IEEE/ ASME Transactions on Mechatronics, vol. 19, no. 1, pp. 374383, 2014.

[13] F. Zhu and R. G. Parker, "Non-linear dynamics of a one-way clutch in belt-pulley systems," Journal of Sound and Vibration, vol. 279, no. 1-2, pp. 285-308, 2005.

[14] H. Ding and D.-P. Li, "Static and dynamic behaviors of beltdrive dynamic systems with a one-way clutch," Nonlinear Dynamics, vol. 78, no. 2, pp. 1553-1575, 2014.

[15] H. Ding, Z. Zhang, and L.-Q. Chen, "Vibration reduction effect of one-way clutch on belt-drive systems," European Journal of Mechanics-A/Solids, vol. 71, pp. 378-385, 2018.

[16] A. Kahraman, "Natural modes of planetary gear trains," Journal of Sound and Vibration, vol. 173, no. 1, pp. 125-130, 1994.

[17] J. Lin and R. G. Parker, "Sensitivity of planetary gear natural frequencies and vibration modes to model parameters," Journal of Sound and Vibration, vol. 228, no. 1, pp. 109-128, 1999.

[18] T. Sun and H. Hu, "Nonlinear dynamics of a planetary gear system with multiple clearances," Mechanism and Machine Theory, vol. 38, no. 12, pp. 1371-1390, 2003.

[19] S. Li, Q. Wu, and Z. Zhang, "Bifurcation and chaos analysis of multistage planetary gear train," Nonlinear Dynamics, vol. 75, no. 1-2, pp. 217-233, 2014.

[20] L. Xiang, N. Gao, and A. Hu, "Dynamic analysis of a planetary gear system with multiple nonlinear parameters," Journal of Computational and Applied Mathematics, vol. 327, pp. 325340, 2018.

[21] C. Gill-Jeong, "Nonlinear behavior analysis of spur gear pairs with a one-way clutch," Journal of Sound and Vibration, vol. 301, no. 3-5, pp. 760-776, 2007.

[22] Z. Liu, H. Yan, Y. Cao, and Y. Lai, "Bifurcation and chaos analysis of the spur gear transmission system for one-way clutch, two-shaft assembly," Shock and Vibration, vol. 2017, Article ID 8621514, 12 pages, 2017.

[23] A. Haris, E. Motato, M. Mohammadpour et al., "On the effect of multiple parallel nonlinear absorbers in palliation of torsional response of automotive drivetrain," International Journal of Non-linear Mechanics, vol. 96, pp. 22-35, 2017.

[24] W.-B. Shangguan, X.-L. Liu, Y. Yin, and S. Rakheja, "Modeling of automotive driveline system for reducing gear rattles," Journal of Sound and Vibration, vol. 416, pp. 136-153, 2018.

[25] X. Tang, X. Hu, W. Yang, and H. Yu, "Novel torsional vibration modeling and assessment of a power-split hybrid electric vehicle equipped with a dual-mass flywheel," IEEE Transactions on Vehicular Technology, vol. 67, no. 3, pp. 1990-2000, 2018.

[26] P. Gao, P. D. Walker, H. Liu, S. Zhou, and C. Xiang, “Application of an adaptive tuned vibration absorber on a dual lay-shaft dual clutch transmission powertrain for vibration reduction," Mechanical Systems and Signal Processing, vol. 121, pp. 725-744, 2019.

[27] $\mathrm{H}$. Wu and G. Wu, "Driveline torsional analysis and clutch damper optimization for reducing gear rattle," Shock and Vibration, vol. 2016, Article ID 8434625, 24 pages, 2016.

[28] G.-W. Kim and S.-C. Shin, "Research on the torque transmissibility of the passive torsional vibration isolator in an automotive clutch damper," Proceedings of the Institution of Mechanical Engineers, Part D: Journal of Automobile Engineering, vol. 229, no. 13, pp. 1840-1847, 2015.

[29] X.-L. Liu, W.-B. Shangguan, X. Jing, and W. Ahmed, "Vibration isolation analysis of clutches based on trouble shooting of vehicle accelerating noise," Journal of Sound and Vibration, vol. 382, pp. 84-99, 2016.

[30] M. Maatar and P. Velex, "An analytical expression for the time-varying contact length in perfect cylindrical gears: some possible applications in gear dynamics," Journal of Mechanical Design, vol. 118, no. 4, pp. 586-589, 1996.

[31] R. G. Parker and J. Lin, "Mesh phasing relationships in planetary and epicyclic gears," Journal of Mechanical Design, vol. 126, no. 2, pp. 365-370, 2004. 